\title{
MarsCAT: Mars Array of ionospheric Research Satellites using the CubeSat Ambipolar Thruster
}

\author{
Edgar A. Bering, III ${ }^{1}$, Lawrence S. Pinsky ${ }^{2}$, Liming $\mathrm{Li}^{3}$, David Jackson ${ }^{4}$, and $\mathrm{Ji}_{\mathrm{Chen}}{ }^{5}$ \\ University of Houston, Houston, Texas, 77204, USA \\ Helen Reed ${ }^{6}$ \\ Texas A \& M University, College Station, Texas, 77843, USA \\ Mark. B. Moldwin ${ }^{7}$, Justin Kasper ${ }^{8}$, J. P. Sheehan ${ }^{9}$, James Forbes ${ }^{10}$, Thomas Heine ${ }^{11}$ \\ University of Michigan, Ann Arbor, Michigan, 48109, USA \\ Anthony Case ${ }^{12}$, Michael Stevens ${ }^{13}$ \\ Harvard/Smithsonian Center for Astrophysics, Cambridge. Mass., 02138, USA \\ and \\ David G. Sibeck ${ }^{14}$ \\ NASA Goddard Space Flight Center, Green Belt, Maryland, 20771, USA
}

\begin{abstract}
The MarsCAT (Mars Array of ionospheric Research Satellites using the CubeSat Ambipolar Thruster) Mission is a two 6U CubeSat mission to study the ionosphere of Mars proposed for the NASA SIMPLeX opportunity. The mission will investigate the plasma and magnetic structure of the Martian ionosphere, including transient plasma structures, magnetic field structure and dynamics, and energetic particle activity. The transit plan calls for a piggy back ride a major mission using a CAT burn for MOI, the first demonstration of CubeSat propulsion for interplanetary travel. MarsCAT will make correlated multipoint studies of the ionosphere and magnetic field of Mars. Specifically, the two spacecraft will make in situ observations of the plasma density, temperature, and convection in the ionosphere of Mars. Multipoint observations are crucial requirments for attaining all the major science goals. They will also make total electron content measurements along the line of sight between the two spacecraft and simultaneous 3-axis local magnetic field measurements in two locations. Additionally, MarsCAT will demonstrate the performance of new CubeSat telemetry antennas designed at the University of Houston that are designed
\end{abstract}

\footnotetext{
${ }^{1}$ Professor, Physics and ECE, 617 Science and Research I, 5005, Associate Fellow.

${ }^{2}$ Professor, Physics, 617 Science and Research I, 5005,

${ }^{3}$ Assistant Professor, Physics, 617 Science and Research I, 5005,

${ }^{4}$ Professor, Electrical and Computer Engineering, N308 Engineering Building I, 4005,.

${ }^{5}$ Professor, Electrical and Computer Engineering, N308 Engineering Building I, 4005,.

${ }^{6}$ Professor, Aerospace Engineering, 3141 TAMU, Fellow.

${ }^{7}$ Professor, Atmospheric, Oceanic and Space Sciences, Space Research Bldg, 2455 Hayward St.,

${ }^{8}$ Associate Professor, Atmospheric, Oceanic and Space Sciences, Space Research Bldg, 2455 Hayward St.,

${ }^{9}$ Asst. Research Scientist, Aerospace Engineering, 1056 FXB 1320 Beal Ave, Member.

${ }^{10}$ Assistant Professor, Aerospace Engineering, FXB 1320 Beal Ave, Member.

11 Graduate Student, Atmospheric, Oceanic and Space Sciences, Space Research Bldg, 2455 Hayward St., Student Member.

${ }^{12}$ Astrophysicist, 60 Garden Street, M/S-58

13 Astrophysicist, 60 Garden Street, M/S-58

${ }^{14}$ Astrophysicist, THEMIS Project Scientist, Code 674.
} 
to be low profile, rugged, and with a higher gain than conventional monopole (whip) antennas. The two MarsCAT CubeSats will have five science instruments: a 3-axis DC magnetometer, adouble-Langmuir probe, a Faraday cup, a solid state energetic particle detector (Science Enhancement Option), and interspacecraft total electron content radio occulation experiment. The MarsCAT spacecraft will be solar powered and equipped with a CAT thruster that can provide up to $4.8 \mathrm{~km} / \mathrm{s}$ of delta- $\mathrm{V}$, which is sufficient to achieve Mars orbit using the Mars 2020 piggyback. They have an active attitude control system, using a sun sensor and flight-proven star tracker for determination, and momentum wheels for 3axis attitude control.

\section{Nomenclature}

$R A A N=$ right ascension of the ascending node

\section{Introduction}

$\mathrm{M}$ ARS has been a source of fascination and wonder for millennia. Quantitative scientific investigation began with the work of Tycho Brahe and Johannes Kepler in the $16^{\text {th }}$ and $17^{\text {th }}$ centuries. In situ investigation of Mars began with the fly-by of Mars by Mariner 4 on 14-15 July 1965, fifty years ago this summer. Since then, humankind has sent 42 missions to Mars, 21 of which have succeeded. The main focus of this fleet of probes has been to understand the geological history of the surface of Mars. By contrast, the latest US orbiter, MAVEN, is the first probe designed to study the Mars' ionosphere ${ }^{1,2,3}$. The MarsCAT mission proposed here will also study the Martian ionosphere as a cohesive multipoint mission. We have proposed the MarsCAT mission to address a set of exciting and important scientific questions that align with and even lie beyond the scope of the MAVEN..

\section{Science Goals}

The MarsCAT mission consists of two 6U CubeSats placed in Mars orbit. It is equipped with magnetometers, Faraday cups, double-Langmuir probes, and an interspacecraft total electron content radio occulation experiment. Together these will measure the magnetic fields, particle density, charge, velocity, and temperature across a range of energies and enable us to achieve the following goals:

- Determine the cause of the density anomalies seen in the Martian ionosphere.

- Quantify the energy balance of the Mars ionosphere

- Determine the response of the ionospheric plasma temperature to solar activity and the solar cycle

- Quantify the role of residual magnetism upon the structure and dynamics of the Martian ionosphere

- Separate spatial from temporal effects to understand the dynamics of minimagnetospheres in the Martian system

- Quantify the physics of reconnection in the weak magnetic field environment of minimagnetospheres

- Determine the role of plasma disturbances such as flux ropes and Kelvin-Helmholtz waves in the solar wind-Martian interaction.

Most exploration of the Martian ionosphere has taken the form of electron density measurements made by either the radio occultation technique ${ }^{4,5}$, or vertical profiles made by topside sounding ${ }^{6,7}$. The retarding potential analyzers (RPAs) on the Viking landers have provided the only ion composition, ion temperature and electron temperature data available ${ }^{8}$. Developing an understanding of Mars' aeronomy is a necessary and important part of unravelling the history of the Martian climate ${ }^{3}$. MAVEN, the first aeronomy mission to Mars, is the first spacecraft to provide simultaneous electron density and temperature, ion density, composition and temperature, and magnetic field data. However, a single mission will not be able to provide sufficient data to answer all open questions about Martian aeronomy. In particular, a single spacecraft cannot separate spatial from temporal variations, nor can it study extended structures in two dimensions. Finally, the primary MAVEN mission is one year, and the most optimistic extended mission scenario lasts 9 years. MarsCAT would arrive sometime after 2021 depending on ride. The timing of arrival might provide data that augments or, more likely, extends observations that are aligned with MAVEN's mission objectives. MarsCAT will address two of the four top Martian aeronomy goals identified in the aeronomy 
white paper submitted to the recent Decadal Survey ${ }^{3}$. It is important to note that full atteainment of all our goals requires multipoint observations.

A. Determine the cause of the density anomalies seen in the Martian ionosphere.

The overall lack of existing data on the Mars ionosphere have precluded development of such basic data/modelling products as maps of the ionosphere. Models predict that the Mars ionosphere should be a simple photochemical Chapman layer 9 . Generally, the observed solar zenith angle dependence confirms this view. However, the limited existing data show anomalies. The ionosphere is not as uniform as expected. There is an enhancement at $90^{\circ} \mathrm{E}$ longitude ${ }^{10}$. The density data exhibit a much larger variance than the models predict ${ }^{11,12}$. Possible explanations include the presence of transport processes, variations in the underlying neutral density and plasma wave processes. More density data are required to enable us to explore the parameter space of possible sources of this variance. The initial phase of the MarsCAT mission will include global mapping observations of electron density made by two spacecraft in nearly circular low Mars orbit. The ability of the MarsCAT spacecraft to work together to measure the total electron content between them will extend and accelerate the mapping process..

\section{B. Quantify the energy balance of the Mars ionosphere}

\section{Determine the response of the ionospheric plasma temperature to solar activity and the solar cycle}

Goals B and C are closely related. The historical data record contains just two single profile temperature measurements of the Martian ionospheric plasma. MAVEN is beginning the task of rectifying this deficiency. MarsCAT will expand this effort, adding many months of data taking to the effort. The mission will map the temperatures globally at altitudes of $180-5000 \mathrm{~km}$ for the entire primary mission. The mission will use "formation flying" in which the two MarsCAT spacecraft will be in elliptical orbits with periareion at $200 \mathrm{~km}$ at mid-latitudes in the Southern hemisphere and apoareion at or above $7000 \mathrm{~km}$. The orbital planes will be separated by a few degrees in RAAN. Using these approaches, we will make both global maps of plasma properties at low altitude and altitude profiles featuring the region of the ionosphere where the most structure is expected.

Ionospheric energy and temperatures are driven by solar EUV radiation input. Our investigation will include correlation studies of the response of the plasma temperatures to solar activity as well as validation studies of ionospheric energy balance models. MarsCAT will provide a unique opportunity to examine the ionospheric energy balance by comparing predicted to observed temperatures and using measured temperature gradients to estimate heat flow. The spaceflight campaign will be conducted in conjunction with a modelling effort that explores the effect of both the solar wind and neutral atmosphere dynamics on the energy balance of the ionosphere

\section{Quantify the role of residual magnetism upon the structure and dynamics of the Martian ionosphere}

Mars Global Surveyor (operating 1997-2006) measured and mapped the vector magnetic field locally at its operating altitude of $378 \mathrm{~km}$ with two 3-axis fluxgate magnetometers ${ }^{13}$. In addition, the MGS used an electron reflectometry technique to infer the total magnetic intensity at $\sim 170 \mathrm{~km}$ altitude ${ }^{14}$. These data show regions of peak magnetic intensity near $180^{\circ} \mathrm{E}$ longitude in the Southern hemisphere. The MarsCAT mission plan will include intervals with periareion at or below $200 \mathrm{~km}$ in this area. The 3-axis MarsCAT magnetometers will enable us to make a vector map of the magnetic field in the high intensity regions. These maps will substantially improve our understanding of the effect these regions have on the structure and dynamics of the ionosphere.

\section{E. Separate spatial from temporal effects to understand the dynamics of minimagnetospheres in the Martian system}

The complex crustal fields observed by MGS have high field regions where the field is so strong that closed "minimagnetospheres" can form ${ }^{14}$. These regions must have some closed field lines. It has been speculated that the outermost field lines can reconnect to the Interplanetary Magnetic Field (IMF) and be opened. Lower altitude $(\sim 200 \mathrm{~km})$ vector measurements by MarsCAT will be used to investigate the validity of this speculation. The plasma in these minimagnetosphere regions should have different dynamics and thermal balance than the surrounding regions. MarsCAT will study the plasma thermal balance and dynamics in these regions by observing electron and ion temperatures, electron density, and ion flow velocity. This goal cannot be achieved at all without simultaneous multipoint measurments. 
MarsCAT will also investigate the magnetosheath density variations predicted by multifluid models in the presence of minimagnetospheres ${ }^{15,16,17}$. According to these models, minimagnetospheres enhance flank sheath densities by factor of 2 . The exception is when draped magnetosheath magnetic fields lie antiparallel to the minimagnetosphere magnetic fields. At these times, reconnection may occur, and create magnetosheath density voids in the regions downstream from the minimagnetospheres.

The presence of minimagnetospheres and reconnection may also account for observations interpreted as indicating that the altitude of the pile-up boundary rises for eastward IMF orientations but falls for southwest IMF orientations when the subsolar latitude lies in the northern hemisphere ${ }^{18}$.

\section{F. Quantify the physics of reconnection in the weak magnetic field environment of minimagnetospheres}

The fact that Mars may have "minimagnetosphere" regions with associated reconnection raises an interesting possibility. One might look for parallel currents, plasma heating and out flows, particle acceleration and even substorms. This question lies in the realm of a fascinating opportunity for exploratory science.

\section{G. Determine the role of plasma disturbances such as flux ropes and Kelvin-Helmholtz waves in the solar wind-Martian interaction.}

The plasma wave spectrum within the Mars ionosphere is largely unknown, though MAVEN's preliminary survey is ongoing ${ }^{19}$. It is clear that a single mission survey will barely begin to catalog and understand either the large scale disturbances or the plasma wave spectrum of the Mars ionosphere. Transient events are common in the vicinity of the ionopause. Their significance in removing the planetary atmosphere ${ }^{20,21}$ depends upon their dimensions, motion, and occurrence rates as a function of solar wind conditions, aspects more readily determined from two-point measurements than from the single point measurements of past missions.

Plasma blobs ${ }^{20,22}$ and magnetic flux ropes ${ }^{23}$ observed in the vicinity of the ionopause have been interpreted in terms of the Kelvin-Helmholtz instability ${ }^{24}$, curvature forces pulling field lines into the ionosphere ${ }^{25}$, and magnetic reconnection, whether between sheared magnetosheath magnetic fields or between sheared magnetosheath ${ }^{26}$ and minimagnetosphere magnetic fields ${ }^{27,28,29}$. Two point measurements will enable to simultaneously monitor the input or boundary conditions for the instabilities that generate these structures and the resulting outflow, e.g., to test for the predicted effects of density gradients ${ }^{30}$ and magnetosheath magnetic field orientations. They will allow us to gauge event dimensions and determine event velocities by timing methods. Minimum variance algorithms applied to the magnetic field measurements will permit determination of boundary orientations ${ }^{31}$. Particle observations within the structures will aid in the determination of magnetic topologies.

In particular, we will use MarsCAT observations to test the criteria governing the Kelvin-Helmholtz instability when wavelike structures are observed, and the likelihood of reconnection when flux ropes are observed. We will seek evidence for the giant plasma flow vortices reported by [32]. Finally, we will test the prediction that the occurrence of the Kelvin-Helmholtz instability is favored in the hemisphere with the outward pointing magnetosheath convection electric field ${ }^{33}$. MarsCAT will make wave observations with the magnetometers, and with the plasma instruments. When the plasma instruments are sweeping, the sweep rate will set the upper frequency limit of the observations. Higher frequency modes will be observed by setting the Langmuir probe and Faraday cup to fixed bias and measuring current collection. The goal is another that cannot be attained without the multipoint observations of MarsCAT.

\section{H. Boundary Interactions}

Previous observations ${ }^{11}$ suggest that the structure of Mars ionosphere cannot be explained by a photochemically formed layer. The interactions between the ionosphere and lower neutral atmosphere probably play important roles in shaping the Mars ionosphere. In addition, solar variations influence Mars ionosphere by modifying the ionospheric energy budget. The proposed global observations of Mars ionosphere by MarsCAT will help us further characterize Mars ionosphere. The modeling effort of three-dimensional (3-D) simulations will help us understand the physics behind the 3-D structure of Mars ionosphere. In addition, newly-developed 3-D simulations of Mars ionosphere that involve the lower neutral atmosphere can investigate the influence of solar activity and atmospheric dynamics (e.g., interaction with the natural atmosphere waves, diffusion, transport) on Mars ionosphere.

Therefore, we propose to include comparative studies between measurements and numerical simulations to further advance understanding of Mars ionosphere. These comparative studies will benefit both the observation and model communities. First, the proposed global measurements will help validate and improve numerical models. 
Second, the full physics and dynamics in the numerical models will help interpret the observations. To be consistent with the proposed global mapping observations, we will use time-dependent 3-D models to simulate Mars ionosphere and its spatio-temporal structures as described in the implementation section.

\section{Measurements To Be Taken}

The two MarsCAT spacecraft have identical instrumentation. They carry an induction Magnetometer (iMAGS) to measure the DC and ULF magnetic wave field (provided by UM), a Faraday Cup Analyzer to measure the electron and ion density, temperature and flow velocity (provided by UM), double-Langmuir Probes (LP) to measure the cold electron plasma density (provided by $\mathrm{UH}$ ) and possibly two Medipix radiation detectors, each with dual orthogonal sensor assemblies to measure the charged particle radiation field and characterize the X-ray bremsstrahlung from below (provide by $\mathrm{UH}$ as part of the SEO options). In addition to these instruments, MarsCAT carries a dual frequency radio package to provide total electron content information between the two spacecraft (provided by $\mathrm{UM}$ ). Both satellites make continuous measurements and return data at various temporal resolutions, although the primary data cadence is 1 second. The quality, temporal resolution and quantity of data from each instrument is discussed in Table 1 ..

\section{A. Data To Be Returned}

The Instrument DPU combines and packetizes the data from the different instruments on board the MarsCAT spacecraft, as shown in Table 1. These data, along with other instrument housekeeping and state of health data are then passed to the spacecraft telemetry system.

\section{B. Quality Of Data To Be Returned}

Table 1: Data Summary

\begin{tabular}{|c|c|c|c|c|c|}
\hline $\begin{array}{l}\text { Instrument } \\
\text { Requirement }\end{array}$ & iMags & $\begin{array}{l}\text { Faraday } \\
\text { Cup (FC) }\end{array}$ & $\begin{array}{l}\text { Langmuir } \\
\text { Probe (LP) }\end{array}$ & $\begin{array}{c}\text { Radio } \\
\text { Sounder (RS) }\end{array}$ & $\begin{array}{c}\text { Radiation } \\
\text { Detector (MP) } \\
\text { (SEO Option) }\end{array}$ \\
\hline Energy Range & $\mathrm{n} / \mathrm{a}$ & $\begin{array}{l}0.1 \mathrm{eV} \text { to } \\
500 \mathrm{eV}\end{array}$ & thermal & thermal & $3 \mathrm{keV}$ to $\mathrm{GeV}$ \\
\hline $\begin{array}{r}\text { Energy } \\
\text { Resolution }\end{array}$ & $\mathrm{n} / \mathrm{a}$ & $18 \%$ & $\pm 100 \mathrm{~K}$ & thermal & $\begin{array}{l}\qquad 1 \mathrm{KeV} \text { for e- } \\
<500 \mathrm{KeV} \\
<\quad 5 \text { ariable } \mathrm{LET}- \\
\text { based for heavy } \\
\text { charged particles }\end{array}$ \\
\hline $\begin{array}{l}\text { Mag Field Res. } \\
\text { (nT) }\end{array}$ & 1 & $\mathrm{n} / \mathrm{a}$ & $\mathrm{n} / \mathrm{a}$ & $\mathrm{n} / \mathrm{a}$ & $\mathrm{n} / \mathrm{a}$ \\
\hline $\begin{array}{cc}\text { Mag } & \text { Field } \\
\text { Range (nT) } & \end{array}$ & $0-10,000$ & $\mathrm{n} / \mathrm{a}$ & $\mathrm{n} / \mathrm{a}$ & $\mathrm{n} / \mathrm{a}$ & $\mathrm{n} / \mathrm{a}$ \\
\hline $\begin{array}{lr}\quad \text { Freq } & \text { Range } \\
\text { (vector/sec } & \text { or } \\
\text { samples/sec) } & \end{array}$ & 16 & $\begin{array}{c}\quad 6.4 \\
\text { samples/s. } \\
32 \text { step sweep }\end{array}$ & $\begin{array}{c}64 \\
\text { samples/s. } 256 \\
\text { step sweep }\end{array}$ & 1 & $\begin{array}{l}\text { Integral } \\
\text { fluences (aka light } \\
\text { curves) } 16 / \mathrm{s} \text {; } \\
\text { energy } \\
3 / \mathrm{s} \quad(4 \quad \mathrm{~min} / \\
\text { spectrum) }\end{array}$ \\
\hline
\end{tabular}




\begin{tabular}{|c|c|c|c|c|c|}
\hline $\begin{array}{c}\text { Time } \\
\text { Resolution (sec) }\end{array}$ & 0.0625 & 5 & 4 & 1 & $\begin{array}{c}.0625 \text { (light } \\
\text { curve) } \\
240 \text { (spectra) }\end{array}$ \\
\hline $\begin{array}{c}\text { Avg Data Rate } \\
\text { (kbps) }\end{array}$ & 1 & 0.04 & 0.5 & 0.25 & 1 \\
\hline
\end{tabular}

\section{Expected Results}

\section{Cause of density anomalies in the Martian ionosphere}

The structure and variability of the Martian ionosphere can only be well determined with simultaneous in situ and remotely sensed observations at different altitudes. MarsCAT will test current models for the Martian Ionosphere. Specifically, the MarsCAT pair will provide the location of the Martian ionopause with concurrent solar wind/magnetosheath density and velocity information throughout the mission. Multipoint MarsCAT observations within the Mars ionosphere will enable us to separate spatial from temporal effects during close spacecraft separation intervals.

The two most likely causes of the observed density anomalies are variations in the neutral atmosphere source and plasma transport. The Faraday cups on both MarsCAT CubeSats will provide the high resolution plasma densities needed to quantify the anomalies, while MarsCAT velocity measurements and density profiles will enable us to assess the role of transport. As described below, modeling will be used to estimate possible changes in background neutral density.

The $\sim 1 / 16 \mathrm{~s}$ cadence of the MarsCAT magnetometer suffices to resolve the structure Martian ionopause [e.g., 31 , while the $\sim 1 \mathrm{~s}$ cadences of all the other instruments suffices to resolve the structure of the broader magnetic pile up region.

2. Quantify the energy balance of the Mars ionosphere

MarsCAT will measure the plasma density, ion and electron temperature, and ion flow velocity profiles at multiple locations in the Martian ionosphere. Other NASA spacecraft at Mars and elsewhere in the solar system will provide solar EUV flux data. If needed, Langmuir Probe photoemission currents can be used to assess the actual EUV flux at MarsCAT. MarsCAT and MAVEN (if still operating) observations will be used to estimate solar wind plasma and magnetic field forcing. Neutral wind input from the lower atmosphere will be estimated using the models discussed below.

These data and model inputs represent a nearly complete assessment of most of the terms in the fluid model energy equation. The balance or lack thereof in this equation will indicate if there are additional sources or sinks to be considered.

3. Determine the response of the ionospheric plasma temperature to solar activity and the solar cycle

By making continuous measurements of the thermal plasma populations in the Martian ionosphere over the lifetime of the mission for a broad range of altitudes, seasons, and solar activity levels, MarsCAT will determine the response of the Martian ionosphere to solar forcing.

4. Quantify the role of residual magnetism upon the structure and dynamics of the Martian ionosphere

MarsCAT will make simultaneous two point measurements of the vector magnetic field along with the density, temperature, and flow velocities within the Martian ionosphere at cadences greater than or equal to $1 \mathrm{~s}$. We will use a combination of direct mapping and assimilative modeling to render and visualize the plasma structures and flow patterns in the vicinity of residual magnetic enhancements. In particular, the data will be examined for evidence of closed flow patterns and regions of temperature and density anomalies.

5. Separate spatial from temporal effects to understand the dynamics of mini-magnetospheres in the Martian system

The separation of spatial from temporal effects is one of the classic problems in experimental space physics. The solution is equally well known, simultaneous observations from multiple spacecraft. MarsCAT is a dual spacecraft mission. The spacecraft orbits have been chosen to provide many simultaneous nearby passes through the likely mini-magnetosphere regions in the Southern Hemisphere of Mars. Spatial structures will recur from orbit to orbit, whereas temporal variations do not. On the other hand, any fluctuations that are seen by both spacecraft in any of the plasma or field parameters are probably signatures of temporal variations. 
$\operatorname{Per}^{32}$ [32], as cited by ${ }^{33}$ [33], potential crustal reconnection regions cover about $7 \%$ of the Martian surface and extend upwards more than $1300 \mathrm{~km}$. Consequently encounters will be relatively common. At $400 \mathrm{~km}$, the maximum radial magnetic field strength associated with any crustal feature is on the order of $220 \mathrm{nT}$. By contrast, fields as large as $1600 \mathrm{nT}$ have been observed at $100 \mathrm{~km}^{13}$. Both values lie comfortably within the range observable by the magnetometers on MarsCAT. Encounters have durations on the order of 5-20 $\mathrm{min}^{34}$ [e.g., 34] and are therefore readily resolvable by all instruments.

6. Quantify the physics of reconnection in the weak magnetic field environment of mini-magnetospheres

Mini-magnetosphere reconnection events are expected to have two possible micro-scale signatures, parallel plasma flows moving at or near the Alfvén speed, and shock accelerated energetic electrons. The Faraday cup will detect high-speed plasma flow in the boundary layers of mini-magnetosphere regions. Reconnection may also result in the detachment of anti-sunward-moving flux ropes or blobs from mini-magnetospheres. MarsCAT will employ its two-point measurements to identify and track these anti-sunward-moving features.

Some of the energetic electron flux coming from reconnection acceleration processes will precipitate into the neutral atmosphere. This precipitation will, among other things, result in the emission of X-Ray bremsstrahlung. The Medipix radiation detectors (part of the Science Enhancement Opportunity options), have a detection threshold for all types of ionizing radiation including X-rays of about $3 \mathrm{KeV}$. These devices can determine the traversal axis of energetic charged particles and the actual direction for stopping particles such as incident electrons $<500 \mathrm{KeV}$. Spectral information for X-Rays, as well as the directional information that can be determined by comparison of the relative fluxes seen by the 4 separate sensors, will enable the inference of locations of X-Rays due to It will be possible, therefore, to remotely sense energetic electron precipitation taking place 100's of $\mathrm{km}$ from the MarsCAT field lines. The X-Ray images delineate the footprints of the region in which reconnection is occurring. The X-Ray energy spectra can be used to infer the spectra of the precipitating electrons that caused the emissions, providing crucial information concerning reconnection.

An encounter with a magnetotail current sheet reconnection region similar to those expected at minimagnetospheres was reported by [34] and lasted some 30-60 s. The $\sim 5 \mathrm{~s}$ time resolution of the MarsCAT plasma and magnetometer instruments will suffice to resolve these regions. Enhanced fluxes of electrons were seen at energies ranging from 10-120 eV. Reconnection is expected to generate outflows with velocities on the order of the Alfvén speed. Taking a mini-magnetosphere magnetic field strength of $40 \mathrm{nT}$ and a magnetosheath density of $\sim 5$ $\mathrm{cm}^{-3}$, outflows on the order of $400 \mathrm{~km} \mathrm{~s}^{-1}$ are expected, within the range of proton velocities observable by the Faraday cup.

7. Determine the role of plasma disturbances such as flux ropes and Kelvin-Helmholtz waves in the solar windMartian interaction.

Instabilities at the Martian ionopause can result in the occurrence of Kelvin-Helmholtz waves and flux ropes. Because they carry away the Martian ionosphere, both may play an important role in the interaction of the solar wind with Mars. The significance of each depends upon the occurrence rates and dimensions of the events they generate as a function of solar wind conditions. When widely separated, the two MarsCAT spacecraft can be used to monitor solar wind drivers and survey ionopause responses simultaneously. When closely-separated, the twopoint measurements will be used to measure the boundary conditions needed to test instability criteria, observe the resulting outflow, and determine event velocities and dimensions. For example, they can be used to test the predicted effects of density gradients ${ }^{30}$ and magnetosheath magnetic field orientations. Minimum variance algorithms applied to the magnetic field measurements will permit determination of boundary orientations ${ }^{31}$. Particle and plasma observations within the structures will aid in the determination of magnetic topologies.

In particular, we will use MarsCAT observations to test the criteria governing the Kelvin-Helmholtz instability when wavelike structures are observed, and the likelihood of reconnection when flux ropes are observed. We will seek evidence for the giant plasma flow vortices reported by ${ }^{35}$. Finally, we will test the prediction that the occurrence of the Kelvin-Helmholtz instability is favored in the hemisphere with the outward pointing magnetosheath convection electric field ${ }^{36}$.

Event durations are on the order of 1 to several minutes ${ }^{29}$, consequently they will be readily resolved by MarsCAT instrumentation. MarsCAT will make wave observations with the magnetometers, and with the plasma instruments. When the plasma instruments are sweeping, the sweep rate will set the upper frequency limit of the observations. Higher frequency modes will be observed by setting the double-Langmuir probe and Faraday cup to fixed bias and measuring current collection.

8. Understand the causes of the infrequent Martian aurora

The recent results from MAVEN have confirmed the occurrence of Martian aurora ${ }^{37}$. Nonetheless, this science objective is an exploratory, relatively high risk goal and is part of the Science Enhancement Opportunity options. As 7

American Institute of Aeronautics and Astronautics 
described above, the Medipix radiation detectors have a detection threshold for all types of ionizing radiation including X-rays of about $3 \mathrm{keV}$. The combination of 4 separately oriented sensors will allow inference of the arrival direction of incoming X-Rays, which in turn will enable the localization of bremsstrahlung emissions from the underlying ionosphere. It should thus be possible to remote sense energetic electron precipitation taking place 100 's of km from the MarsCAT field lines. The X-Ray energy spectra can then be used to infer the spectra of the precipitating electrons that caused the emissions.

\section{Science Products And Data}

The MarsCAT instruments provide a variety of data products to be used for scientific analysis. The MarsCAT team includes co-investigators who use the data for both case study and statistical analysis of the controlling factors for ionosphere, ionopause and mini-magnetosphere variability; ingest into models of the Martian atmosphere; and for testing theories of wave-induced particle acceleration and loss. The results of these studies will be presented at scientific meetings and published in the peer-reviewed scientific literature.

\section{Modeling the Lower Boundary}

Three-dimensional simulation of Mars' ionosphere is a rapidly evolving research area ${ }^{38,39}$. As part of the MarsCAT effort, we will couple a photochemistry model with a general circulation model (GCM) to conduct 3-D simulations of the Martian ionosphere. For the photochemistry model, we will use the Caltech/JPL multidimensional model (KINETICS), that was built to answer fundamental questions concerning the chemical composition and dynamical processes responsible for the photochemistry and the transport/mixing of chemical species in planetary atmospheres [e.g. 9,40,41,42,43]. The Caltech/JPL KINETICS model is generally run in onedimensional (1-D) $)^{41}$ and two-dimensional (2-D) modes $^{42,44}$ to save computer power and time. However, the model has the structure to run 3-D simulations. For 3-D simulations, the model needs dynamic output from a GCM simulation. Therefore, dynamic variables (e.g., winds and mixing coefficients) from the GCM simulation will be used to drive the 3-D photochemistry model.

The planetWRF Mars GCM $^{45,46}$ will be used in the coupling. PlanetWRF is a flexible planetary atmospheric numerical model which has the ability to simulate the atmospheric dynamics of different planets at multiple scales. This model, which is an enhancement and generalization of the terrestrial Weather Research and Forecasting (WRF) model developed by the National Center for Atmospheric Research (NCAR), has been converted for use on Mars by the addition of Mars-specific parameterizations, such as radiation, orbital mechanics, and a $\mathrm{CO}_{2}$ sublimation cycle.

Coupling between the Caltech/JPL KINETICS model and the planetWRF model will make it possible to simulate the time-independent 3-D structure of Mars ionosphere, investigate the roles of solar activities in reshaping Mars ionosphere, and explore the interaction between Mars ionosphere and the lower neutral atmosphere. Comparative study of the 3-D simulation results and the proposed global observations from this mission will further advance our understanding of Mars ionosphere. In particular, the coupled models will be used to study the possible roles of atmospheric dynamics in the variable, structured, electron density in Mars ionosphere revealed by the observations from the Mars Global Surveyor ${ }^{11}$.

\section{Ionopause studies}

Ion pickup may be a major loss process for the Martian atmosphere. Pickup occurs when and where charges solar wind ions encounter neutral exospheric atoms. Because the density of neutral atoms falls off rapidly with distance from the planet, ion pickup increases greatly when the ionopause moves inward toward the planet. Determining the location, structure, and motion of the ionopause is consequently an important task, and one that is greatly facilitated by the presence of two identically-equipped spacecraft.

According to [30], the ionopause transition between plasmas and magnetic fields of solar wind versus planetary origin generally occurs some 300 to $600 \mathrm{~km}$ above the surface of the planet and can be either gradual or sharp (for reasons that remain unknown). Two-point MARSCAT plasma and magnetic field measurements will be used to map the locations of the ionopause. When the spacecraft are nearby, the two point measurements can be used to determine ionopause velocities and (assuming simple radial advection) the fine structure of this boundary. For example, there is evidence indicating lumpy or permeable structures near radial crustal remnant magnetic fields ${ }^{18}$. The simple advection hypothesis can be tested by checking whether both spacecraft observe corresponding structures.

Increases in the solar wind dynamic pressure may ${ }^{18,47,48}$ or may not ${ }^{49}$ move the magnetic pile-up and photoelectron boundaries at the ionopause towards the planet. The presence of hot flow anomalies upstream from the planet may create transient enhancements and decreases in the pressure applied to the ionopause, resulting in 
large amplitude motion [e.g. 50]. When interspacecraft separation distances between the spacecraft are large, one will be used to monitor incoming magnetosheath conditions and the other to determine the location, motion, and structure of the ionopause as a function of these conditions. We will test whether ${ }^{18}$ or not $^{31,51}$ the boundary moves significantly in response to variations in solar EUV. We will inspect the evidence indicating that crustal magnetic fields corresponding to minimagnetospheres raise the altitude of the magnetic pileup boundary (i.e., ionopause) ${ }^{27}$ or even replace it, as predicted by simulations ${ }^{15,16}$.

With appropriate models for charge exchange and the exosphere, we will then determine atmospheric loss rates as a function of solar wind conditions, e.g. during the space weather storms when it is expected to increase ${ }^{52,53}$, solar EUV, and in the presence of crustal structures.

\section{E. Data Analysis Plan}

The MarsCAT data management plan integrates the in situ plasma and magnetometer measurements and the remote sensing radio observations - from both spacecraft - into a single coherent web-based database that provides not only quick-look plotting but also detailed analysis capabilities directly over the Internet. This data management model allows scientists from both within the MarsCAT team and from the broader Mars scientific community access to all the high-resolution data. We will use the THEMIS Space Physics Environment Data Analysis Software (http://themis.igpp.ucla.edu/software.shtml), which is written in IDL. The web interface is customizable allowing for quick look plots of specific intervals from specific instruments and spacecraft, analysis of correlation between different spacecraft measurements, and the ability to download the data in various formats. The open data architecture and innovative integrated database allows for the maximum scientific return by providing meaningful access to the data to the entire solar-terrestrial physics community. It is being used by a variety of NASA missions.

\section{Mission Implementation}

\section{A. Mission Objectives}

The primary objectives of the MarsCAT mission are as follows:

1) Explore the interaction between Mars' atmosphere and the solar wind as defined by the MarsCAT science goals.

2) Demonstrate CubeSat interplanetary exploration enabled by the CubeSat Ambipolar Thruster (CAT).

3) Support, augment, and possibly extend the mission objectives of NASA's MAVEN.

The following sections discuss the feasibility of meeting these objectives.

\section{B. Concept of Operations}

MarsCAT consists of two identical 6U CubeSat spacecraft compliant with applicable Cal Poly CubeSat and Poly Picosat Orbital Deployer (PPOD) standards ${ }^{54}$, and with the paper An Advanced Standard for CubeSats, ${ }^{55}$, related to 6U and larger CubeSats, and with NASA SMD Guidelines For Heliophysics EM-1 CubeSat Proposers, [2014].

The authors have determined that the best option for the MarsCAT spacecraft is to launch piggyback on a major mission and remain stowed onboard until reaching Mars. They will jettison from the mission carrier at the edge of Mars' gravitational sphere of influence and use the CAT to achieve capture and Mars orbital insertion (MOI). Exhaustive trades revealed three compelling reasons that made the EM-1 opportunity (with release at Earth escape velocity) infeasible. First, even with the CAT propulsion system, the amount of propellant required for independent transit to Mars by each spacecraft, followed by MOI to a relevant science orbit, would be prohibitive given the constraints of a $6 \mathrm{U}$ form factor. Second, it was deemed that the risk of both spacecraft navigating a transit trajectory to Mars independently and reliably arriving within a reasonable time window for multipoint science observations was too high. Third, mitigating the risk of additional radiation exposure on an independent transit (without the shielding mass of a pod carrier) was infeasible within the available $6 \mathrm{U}$ mass constraint. For these reasons, MarsCAT proposes to launch with a major mission.

Each MarsCAT spacecraft is equipped with the CubeSat Ambipolar Thruster (CAT), a 3-axis attitude determination and control system capable of attitude solutions in interplanetary space and near Mars, and an embedded radio navigation system utilizing the navigation capabilities of the Mars Relay's CCSDS protocol. Upon ejection from Mars 2020 at Mars' gravitational sphere of influence (500,000 km altitude), the CAT performs a finite continuous maneuver to transition from MOI to a highly elliptical, stable orbit of $200 \times 7000 \mathrm{~km}$ and $60^{\circ}+/ 5^{\circ}$ inclination. The planes of the two MarsCAT orbits will be separated by approximately $15^{\circ}$ in right ascension of the ascending node (RAAN)..

9

American Institute of Aeronautics and Astronautics 
Each MarsCAT spacecraft carries a science payload consisting of two three-axis magnetometers, a Faraday cup, double-Langmuir probes, and a dual-frequency crosslink radio occultation experiment embedded within the spacecraft communication system. Communications with Earth will be over UHF via the Mars Relay Electra package on MAVEN and MRO. MarsCAT operations are planned for one year following Mars orbit insertion.

During the disposal phase, any remaining fuel will be used to raise the periapsis as high as possible. This requires very little fuel because of the eccentricity of the orbit and changes of $100 \mathrm{~km}$ can be accomplished with only $\sim 10 \mathrm{~m} / \mathrm{s}$ delta-v expended at apoapsis. Preliminary analysis shows MarsCAT complies with the COSPAR Planetary Protection Policy, [2011] and NASA Procedural Requirement NPR 8020.12D, Planetary Protection for Robotic Extraterrestrial Spacecraft [2011]. The planetary protection requirement is a disposal orbit with 0.99

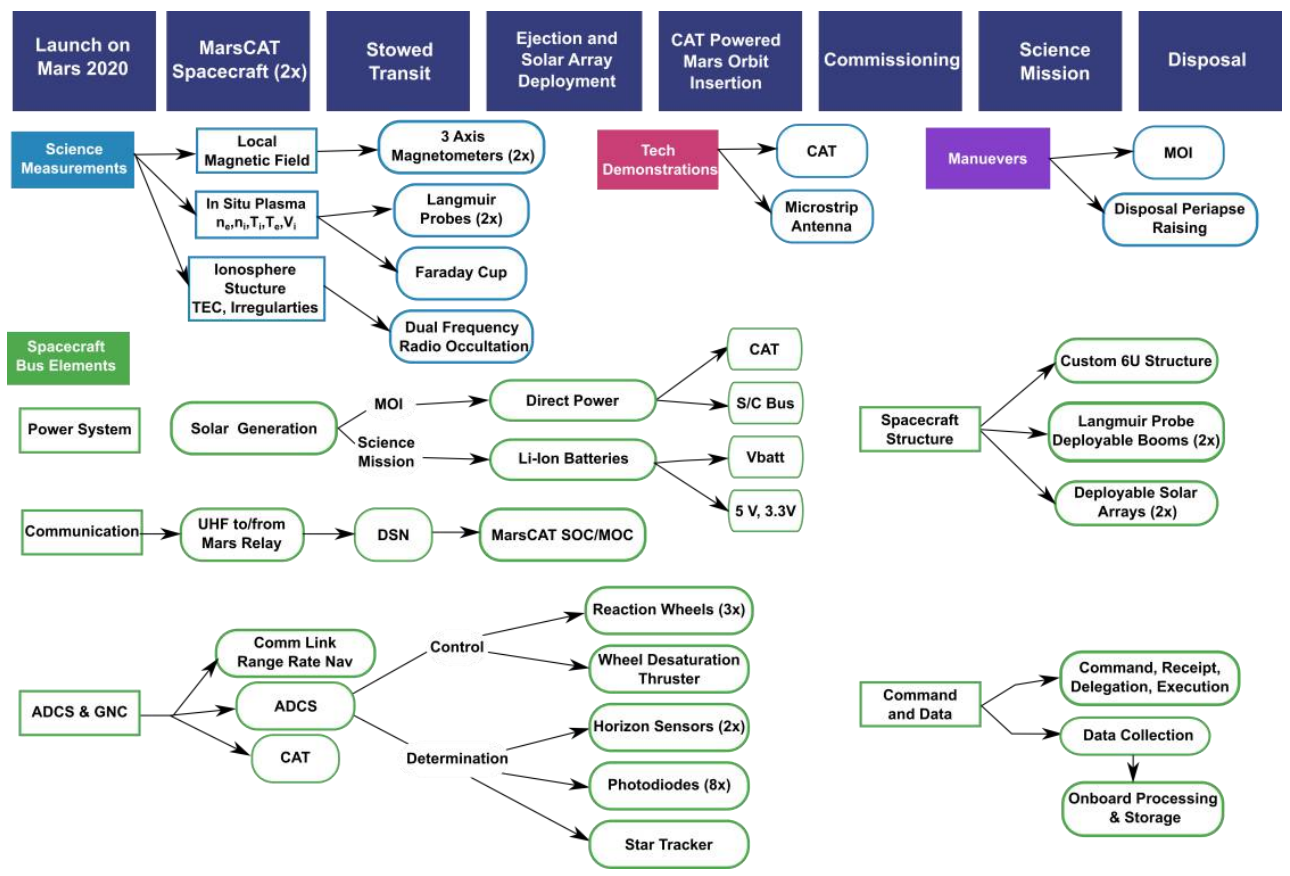

certainty of no impact with Mars within 20 years and 0.95 certainty within 50 years.

\section{Figure 1: MarsCAT Concept of Operations}

\section{Launch \& Mars Transit}

Two launch and Mars transfer scenarios were analyzed: 1) Deploy from EM-1 and perform transfer maneuvers using the CAT; 2) Launch on a podcarrier riding wit a larger mission and deploy when nearing Mars and use CAT to slow for MOI. Analysis showed option 1 (launching on EM-1) would not be feasible to complete mission objectives; the delta-v required from Earth escape velocity to Mars transfer would leave insufficient propellant to lower into an operational orbit. Starting from Earth escape (EM-1 release) and assuming a best-case scenario that the two 6Us were released in the ideal direction and no pointing adjustments were necessary, $3.38 \mathrm{~km} / \mathrm{s}$ of delta-v would be required for each spacecraft for trans-Mars injection. [A lower delta-v of $2.98 \mathrm{~km} / \mathrm{s}$ could be possible, however, this is only achieved by spreading the maneuver over multiple orbits of the sun, in essence becoming like a Hohmann transfer spread over many orbits. Each additional orbit adds $>1$ year to the voyage, and it would take many orbits to approach that best case delta-v, which is not practical and very risky given both that there are two spacecraft involved and the extended exposure to the radiation environment.] Assuming $6 \mathrm{~kg}$ of iodine propellant (50\% of the total mass) and an Isp of 690s, each spacecraft would have $4.78 \mathrm{~km} / \mathrm{s}$ of delta-v capacity, so that $1.40 \mathrm{~km} / \mathrm{s}$ would then be left to capture into a Mars orbit. The calculations show that this is insufficient to achieve any orbit around Mars. At a minimum, about $1.8 \mathrm{~km} / \mathrm{s}$ of delta-v would be required to achieve a non-circular orbit around Mars, however, the capture orbit would be too highly eccentric, with an apoapsis near the edge of Mars' sphere of influence and very little time spent at low altitude for the science.

Based on these trades, the team concluded that the only feasible option to meet the science and end of life requirements was to go with option 2, and piggyback on a larger mission to a Mars transfer orbit and deploy when

10

American Institute of Aeronautics and Astronautics 
nearing Mars and use CAT to slow for MOI. The balance of this document assumes option 2 as an element of the concept of operations.

\section{Science Instruments Overview}

The structure and dynamics of the plasma and magnetic field environment around Mars is determined by interaction of solar wind and the Martian ionosphere and crustal magnetic field. Therefore, four instruments are needed to determine the local solar wind and Mars ionospheric properties and to address all of the science requirements: a magnetometer, two plasma experiments, and a remote sensing radio experiment. The investigators were selected based on their strong expertise and experience in instrumentation for space missions. The instruments share a common spacecraft bus interface, spacecraft DPU, and ground system operations.

\section{Magnetometer}

The Induction Magnetometer (iMAG) to be flown on the two MarsCAT spacecraft is a new magnetometer design, but derives heritage from a long line of space-qualified fluxgate magnetometers built by the Institute of Geophysics and Planetary Physics at UCLA.

Important assets of the iMAG magnetometer are its inherent linearity (with nonlinearities significantly smaller than 1 part in $10^{4}$ ) and the reliability and stability of the gains and offsets. This instrument, which is a completely different design from the traditional fluxgate magnetometer design, is inherently rad hard, low mass, low power and temperature insensitive. The electronics functional block diagram is shown in Figure 2. There is no microprocessor in this simple design.

The iMAGS magnetometer continues the digitization and miniaturization path of the UCLA NASA ST5 and MMS fluxgate magnetometer designs. The current circuit design realizes significant reductions in power, mass and size. Additional mass savings have also been achieved by the use of extremely small sensors and the elimination of the boom and sensor boom cable. Figure 2 shows an engineering version of the iMAG sensor.

The current generation of fluxgate magnetometers being flown on missions such as ACE, Wind, Cluster II are too bulky (several $\mathrm{kg}$ ) and power hungry (several W) to be flown on CubeSat class missions due to the ultraconstrained resource environment of micro-satellites. They are also too expensive. Even more modern small magnetometers, such as built for the NASA ST-5 and MMS missions are large compared to what is proposed here (Table 2).

To address the science requirements, our goal is to reduce the mass and power of DC magnetometers by an order of magnitude over those currently flown while approaching the precision, noise-level, linearity, and stability of past fluxgate magnetometers. An innovative approach enabled by low-cost magnetometers is the elimination of large booms, by placing multiple sensors in and on the bus in combination with a careful magnetic cleanliness program.

Table 2: Comparison between iMAGS and ST-5 magnetometer.

\begin{tabular}{|l|l|l|l|l|l|l|}
\hline Mission Name & Range & $\begin{array}{l}\text { Cadence } \\
{[\mathbf{H z}]}\end{array}$ & $\begin{array}{c}\text { Sensor } \\
\text { Mass } \\
\mathbf{[ g}]\end{array}$ & $\begin{array}{c}\text { Elect. } \\
\text { Mass } \\
{[\mathbf{g}]}\end{array}$ & $\begin{array}{l}\text { Circuit Area } \\
{\left[\mathbf{i n}^{2}\right]}\end{array}$ & $\begin{array}{l}\text { Power } \\
{[\mathbf{W}]}\end{array}$ \\
\hline ST5 & 60,000 & 16 & 75 & 650 & 35 & 0.5 \\
\hline Proposed New Mag & 60,000 & Up to 147 & 15 & 100 & 1 & 0.1 \\
\hline
\end{tabular}

The UM new digital induction magnetometer takes advantage of mobile phone magnetometer sensor development to reduce the mass, power consumption, and increase the radiation tolerance of the fluxgate magnetometer. The instrument does not use an A/D converter making it much more radiation tolerant than traditional fluxgate magnetometer designs. The commercial magneto-inductive magnetometer from PNI will be used with custom built sensors to increase the sensitivity and a custom housing to provide thermal and radiation protection. In the new induction magnetometer, the magnetic field is measured by counting the time between flips of the magnetic induction of the circuit which is dependent on the strength of the applied DC field ${ }^{56}$. As seen in the functional diagram in Eigure 2 left. the magnetometer is a LR circuit with a Schmitt Trigger for counting pulses.

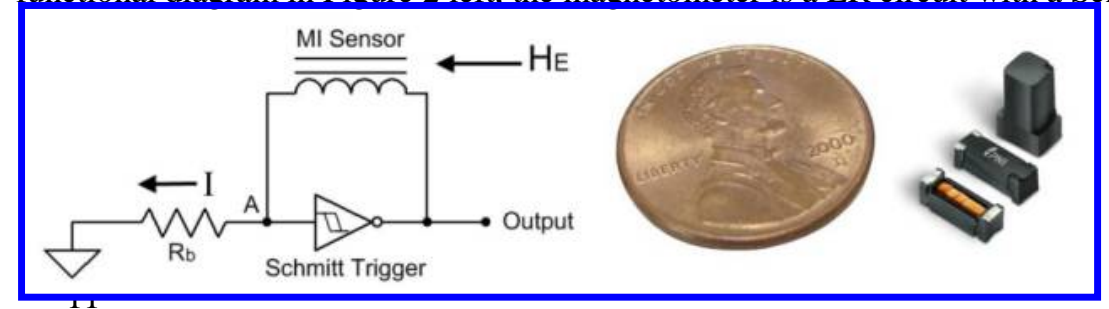

Figure 2 (left) Block diagram of PNI Induction 3-axis UM Magnetometer. (right) Sensor assembly size compared to penny. 
In Figure 2, $\mathrm{H}_{\mathrm{E}}$ is the external magnetic field parallel to the coil. The total field that the sensor experiences is due to the external field and the field generated by the circuit $\left(\mathrm{H}=\mathrm{kI}+\mathrm{H}_{\mathrm{E}}\right.$, where $\mathrm{k}$ is a property of the sensor, and $\mathrm{I}$ is the current through the circuit). The Schmitt trigger causes the current through the circuit to oscillate as the voltage bases a set "trigger" value. The time between oscillations or trigger flips is dependent on the strength of the external field and therefore the DC field can be measured by simply counting the number of flips or triggers. This design is inherently radiation tolerant due to the elimination of the need for any form of ADC. Power consumption and mass are lowered by a reduction in the number of amplifiers required, as well as the elimination of the ADC. In fact the entire three-axis magnetometer can be implemented on one $1 \mathrm{~cm} \times 2 \mathrm{~cm}$ printed circuit board.

The size and mass of the new magnetometer instrument is extremely modest compared to traditional fluxgate magnetometers and the design significantly reduces the number of components in the overall analog circuitry. The prototype vector iMAGs magnetometer weighs less than $10 \mathrm{gm}$ including the custom built sensors, but not including the housing.

One hidden cost of fluxgate magnetometers is the need for a boom. This need drives up mission complexity and adds significant mass due to meters of cabling and the boom itself. By driving down the resource needs and cost of the magnetometer, a new approach can be incorporated in MarsCAT that eliminates the need for a boom ${ }^{57,58}$. This approach places several magnetometers inside and on the bus to be able to identify spacecraft magnetic signals in the data so that the external field can be recovered with processing and careful magnetic cleanliness and characterization prior to launch. With CubeSats (even 6U structures), testing the fully integrated system is possible within the UM magnetic testing facilities. Such a characterization and testing was done with the 3 U RAX mission.

The development and testing of the new sensor is on-going (performance testing, environmental testing including radiation and thermal vacuum testing) and a high-altitude balloon flight is scheduled for May 2015. The system is being proposed to fly on Earth orbiting CubeSat missions years prior to the Mars 2020 launch to raise the TRL, provide heritage and reduce the risk on both the sensor and boom-less design.

2. Plasma Faraday Cup Analyzer

Faraday Cup Background. The Faraday Cup (FC) is a heritage plasma sensor and a workhorse for space plasma measurements since the start of space exploration ${ }^{59,60}$. It is the ideal instrument to make plasma density and flow velocity measurements. The Smithsonian Astrophysical Observatory (SAO) and the University of Michigan (UM) are currently working on two FC flight developments: the DSCOVR FC, launched in February 2015, and the SPP SPC that passed PDR, with CDR scheduled for April 2015. FCs on board the Voyager $1 \& 2$ spacecraft provided measurements throughout the solar system, including the solar wind and magnetospheric measurements at Jupiter. Since the sensor is simply a metal plate, the response of the instrument is stable with time ${ }^{61}$ [Kasper et al. 2006]. The FCs on Voyager 2 (launched 1977) and Wind (launched 1994) are still operating and providing invaluable solar wind measurements.

The Faraday Cups (FCs) provide direct observations of the Martian ionospheric plasma. FC instrumentation was selected because it is not sensitive to external noise sources such as penetrating radiation, solar UV, temperature extremes, or radar emission, it requires no further technology development, and it has extensive heritage stretching back to the beginnings of the space age. The FC sensors are more capable in terms of field-of-view (FOV), energy range, sensitivity, and cadence than the similar Voyager Plasma Science Instrument (V-PLS) FC instruments that have operated successfully throughout the solar system.

FC Implementation: Building on previous FC designs (V-PLS and Solar Probe Cup (SPP-SPC)), and informed by Voyager and Galileo observations, the FC design is optimized for characterizing the Martian plasma environment. The FC sensors are simple, inert metal plates that collect currents from ions and electrons. They contain no silicon or other material that would be degraded or damaged by ionizing radiation, making them ideal instruments for precise, stable, and sensitive plasma measurement around Mars. A synchronous detection technique that has been employed for over half a century in similar FC instruments eliminate signals at all frequencies other than the oscillation frequency of an applied HV waveform, making the instrument insensitive to noise sources such as ionization radiation, UV light, or emission generated by other instruments (e.g. radars). The FC preamplifier electronics are a direct descendent of the SPP-SPC electronics, and the high-voltage amplifier is based on previous designs from the University of Michigan.

The FC has a FOV of $90^{\circ}$, measuring the 1.5-dimensional velocity distribution function (VDF; a 1-D reduced distribution function plus vector flow angles as a function of energy/charge) of ions and electrons. FCs measure the current produced on metal collector plates by charged particles sufficient energy per charge (E/q) to pass through a modulated retarding grid placed at variable (AC) high voltage (HV).

In any one measurement, a HV waveform consisting of a DC level $\mathrm{V}$ plus a sine wave with amplitude $\Delta \mathrm{V}$ that sets the energy resolution is applied to the modulator grid. Particles with $\mathrm{E} / \mathrm{q}>\mathrm{V}+\Delta \mathrm{V}$ always make it through the 12

American Institute of Aeronautics and Astronautics 
modulator, producing a constant current. Particles with $\mathrm{E} / \mathrm{q}<\mathrm{V}-\Delta \mathrm{V}$ are reflected out of the sensor. Particles with $\mathrm{V}$ $\Delta \mathrm{V}<\mathrm{E} / \mathrm{q}<\mathrm{V}+\Delta \mathrm{V}$ produce an $\mathrm{AC}$ current. Electronics within the instrument amplify and digitize the current waveform from each collector plate, and then perform a synchronous detection to lock in on only the AC component of the current at the HV modulation frequency. This lock-in process makes the FC insensitive to noise sources such as ionizing radiation, UV light, or a radar instrument on the spacecraft, and has been demonstrated in-flight.

The sum of the currents from all collector plate section gives the particle flux, and the ratios of the currents on each collector plate section give the precise flow angle of the plasma. Since the currents are measured with a very high signal to noise, the instrument is capable of measuring the flow angle with better than $1^{\circ}$ resolution. A full FC measurement cycle consists of stepping $\mathrm{V}$ on the modulator from low voltages (TBD) up to $2 \mathrm{kV}$ to modulate ions. The E/q range scanned by the instrument is set by the range of voltage $\mathrm{V}$ applied. The energy resolution of the instrument is determined by selecting the value of $\Delta \mathrm{V}$; simply changing the amplitude of the AC component of the waveform changes the FC energy resolution on the fly.

Each FC is built from two machined cylindrical housings, each containing a set of metal grids and insulating spacers. Grids are either connected to the chassis (ground) or driven to a voltage by a power supply. The 'modulator grid' is driven with a high-voltage sinusoidal waveform to modulate the flow of particles. The 'suppressor grid' is placed directly above the collector plates and held at a constant $-55 \mathrm{~V}$ to ensure that the measured current is due only to incoming particles and not secondary electrons being ejected from the collector plates.

The signal from each collector plate is connected to its own preamplifier circuit. The preamplifier board converts the input sinusoidal current waveform to a voltage, amplifies that voltage with 3 parallel gain stages, and digitizes the sinusoidal waveform. An FPGA onboard the preamplifier board performs the lock-in algorithm and distributes CCSDS packets containing the amplitude of the waveform at the modulation frequency on each of the collector plates.

The FPGA on the preamplifier board drives digital-to-analog-converters (DACs) that supply the control waveform to the high-voltage power supply (HVPS). In this way, the control waveform is created with precisely the same frequency as the reference waveform used in the lock-in algorithm in the FPGA.

FC Data Requirements: Spectra are acquired every 5 seconds, and consist of measurements in 32 separate E/q windows. 16-bit measurements are returned for each of the 3 collector plates. Thus each spectrum consists of 192 bytes/spectrum, for a total of 3.2 megabytes (MB) per 24-hour period. Including housekeeping and other configuration information, the total telemetry required for full FC operation is $3.8 \mathrm{MB}$ per day. Data fitting techniques will be used where needed to reduce this requirement.

\section{Langmuir Probe}

Double-Langmuir probes (DLPs) measure the ambient ion density, ion flux, and electron temperature, and electron density ${ }^{62,63}$. These probes are not to be confused with "double probes," which is a common term in the space physics community to refer to two boom-mounted probes for making DC-AC electric field measurements. Each probe of the DLP is biased with an AC voltage with respect to the other probe, so that ion or electron loss area problems are mitigated from normal single Langmuir probe problems onboard spacecraft. This means that the entire spacecraft body could be made of insulating material and the DLP would still function nominally. This advantage of DLPs is important for use on CubeSats, since the exposed conducting surface area can be somewhat small. For a single Langmuir probe to provide accurate results, the spacecraft conducting surface area needs to be sufficiently large as an ion loss area when the single Langmuir probe is biased to electron saturation so that the probe itself doesn't alter the ambient space plasma potential that is being measured. For atomic oxygen, this ratio of electron loss area at the probe tip to ion loss area at the spacecraft surface is $\sim 200: 1$, and $250: 1$ for $\mathrm{NO}^{+, 57,64}$. The DLP however, is a floating system and electrically isolated from the spacecraft ground/skin. Ion and electron saturation currents that are drawn to the DLP probe tips are effectively drawn between each probe tip, and the electron saturation current drawn in one probe is limited by the ion saturation current drawn in the other probe. A voltage sweep is required for DLPs, as is a transimpedance (current to voltage) amplifier for measuring the current between the two probes of the DLP. The anticipated ion current collected by each probe is on the order of $\mathrm{nA} / \mathrm{cm}^{2}$, well within the detection limits and signal to noise ratios for modern transimpedance amplifier designs.

DLPs have not been extensively used in the early decades of spaceflight owing to the low ion saturation current densities in orbit and the required current detection limits of the then existing electronics. The modern transimpedance amplifier circuits for this application are non-trivial, but are achievable within the form factor and power limitations of a CubeSat. Up until now, the majority of probes flown into space have been used to measure AC or DC electric fields, though the use of swept single Langmuir probes (SLPs) has been steadily growing ${ }^{57,65}$. Until the advent of automatic gain control circuits, the single Langmuir probe analysis ignored the ion saturation current portion of the I-V trace, Fig. 3. Photoemission from the spacecraft body is the other major complicating 13 
factor. In some cases, the photoemission current can be of a similar current density and can swamp the ion current, or it can lead to measurements of the electron temperature that are too high. The solution requires that the double probes be mounted on extension booms that are far enough away from the spacecraft body. The two probes also need to be separated by several/many Debye lengths from each other so that the ion sheaths do not overlap. However, in the relatively high plasma density regions of low Mars orbit, these issues can be mitigated with careful design. This may limit the effective measurement range of a CubeSat mounted double probe to the interior of the ionosphere.
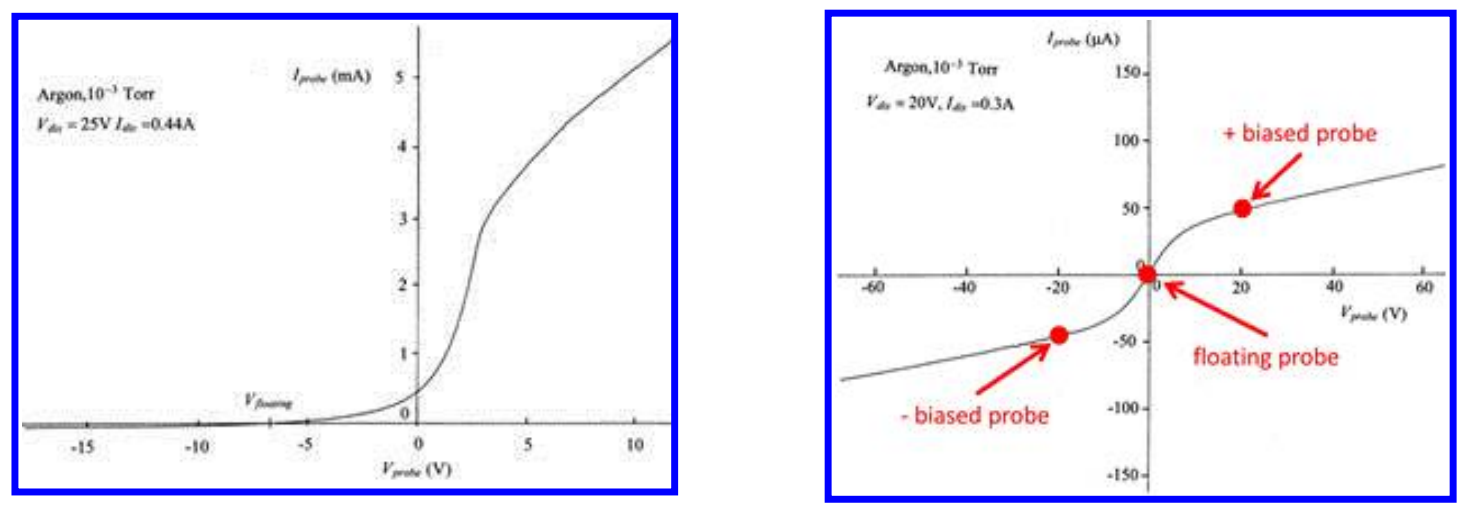

Figure 3. Current-voltage characteristic trace: a) "normal" lab based single Langmuir probe (SLP). b) lab based double Langmuir probe (DLP). Figure adapted from Stenzel, [1997].

Once an I-V characteristic is generated, the plasma parameters can be found from fitting a hyperbolic tangent function. Fig. 3 shows a representative I-V trace from a laboratory based "normal" single Langmuir probe, a), and a laboratory based DLP, b). DLPs are swept in voltage to find the current-voltage characteristic. The power and

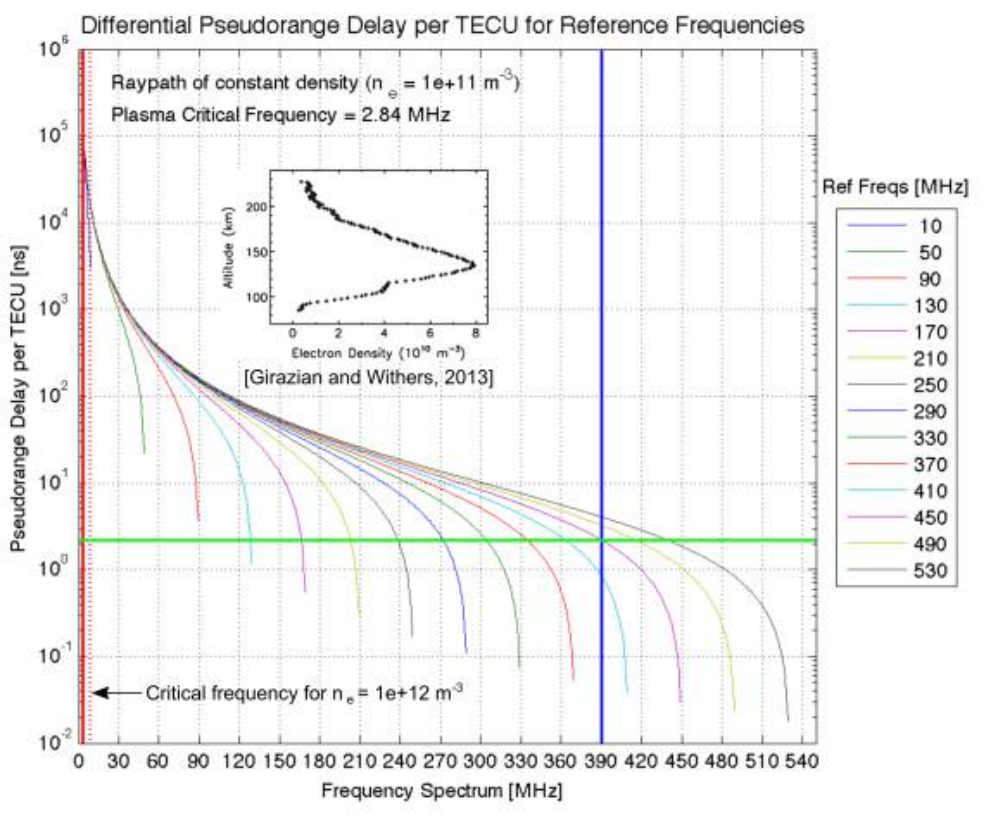

Figure 4. Differential pseudorange delays due to a given plasma density of $1 \mathrm{e} 11$ m-3 along signal raypath. Differential delay between 390 and $450 \mathrm{MHz}$ is 2.2 ns/TECU compared to $1 \mathrm{~ns} / \mathrm{TECU}$ for typical measurements made at Earth using GPS. Solid and dashed red lines show selected frequencies are well above critical frequency for $1 \mathrm{e} 11 \mathrm{~m}^{-3}$ plasma density and also for one order magnitude higher at $1 \mathrm{e} 12 \mathrm{~m}^{-3}$

14

American Institute of Aeronautics and Astronautics volume requirements will be similar to the Langmuir probes on DICE $^{66}$. The probes will deploy on a pair of rigid booms in a "V" configuration to open the path between the probes. Plasms density can then be inferred from observation of the plasma frequency.

4. Dual-Frequency Crosslink Radio Occultation Experiment

Radio tomography of the Earth's ionosphere ${ }^{67}$ has enabled near-global observations of the structure and dynamics of key ionospheric regions and processes. Radio tomography of the Earth's magnetosphere ${ }^{68}$ has also been proposed to help place multipoint in situ observations into global context. MarsCAT will use its telemetry transceivers to measure electron column density data between the two spacecraft, indicative of kilometer-scale density structure. A $10 \mathrm{~s}$ data cadence will provide 1000 s of ray-paths 
per orbit from a variety of viewing geometries including occultation geometries near Mars' periapsis.

The radio science technique of measuring the integrated column electron density (or Total Electron Content (TEC)) is accomplished by having a spacecraft transmit coherently phased pairs of radio frequencies to a receiver. The dispersion of the two frequencies through the Martian ionosphere separates their arrival time at the receiver. The differential delay between the two signals gives an accurate measure of the TEC. At Earth, this can be achieved using dual-frequency GPS signals. However at Mars, the experiment will use the telemetry transceivers on both MarsCAT spacecraft, each will transmit and receive simultaneously at 390 and $450 \mathrm{MHz}$, frequencies selected to maximize dispersion and reduce the time synchronization burden on the receiver oscillator and microprocessor while also remaining with the frequency band of the Mars Relay CCSDS protocol. The two spacecraft clocks will be synchronized using the microprocessor to align a pseudorandom code sequence, similar to how low-precision GPS receivers maintain accurate time.

\section{E. Science Mission Profile}

Once released from the carrier mission into a Mars transfer orbit and using its CAT engine with nominal parameters of $1.1 \mathrm{mN}$ of thrust and approximately $6 \mathrm{~kg}$ of iodine propellant at $690 \mathrm{~s}$ of Isp, each CubeSat inserts into a highly elliptical Martian orbit to perform the science mission and finally raise the periapis for a stable disposal orbit. The orbit inclination is $60^{\circ}+/ 5^{\circ}$ to focus on the concentration of crustal magnetic fields in the southern hemisphere centered around $60^{\circ}$ south latitude and $180^{\circ}$ longitude. A science mission lifetime of 1 Earth year is planned.

Approaching Mars on an impulsive Hohmann trajectory via a larger carrier mission will give the spacecraft an intercept velocity of $2.67 \mathrm{~km} / \mathrm{s}$. While still far from Mars, the trajectory will be adjusted to insert into the desired inclination and periapsis at minimal delta-v cost. The minimum delta-v for Mars capture to an orbit of $200 \times 500,000$ $\mathrm{km}$ would be $2.40 \mathrm{~km} / \mathrm{s}$, costing $3.64 \mathrm{~kg}$ of propellant (assuming an initial wet mass of $12 \mathrm{~kg}$ ). The periapsis altitude has a negligible effect on the delta-v cost, it is lowering the apoapsis that is costly. This capture maneuver is a continuous burn over 4.7 months so as to be captured as quickly as possible.

Once captured, the apoapsis is lowered to decrease the orbital period and increase the total time the spacecraft spends near periapsis, where the science mission is to be completed. The closest orbit achievable will be $200 \times 7,000$ $\mathrm{km}$ which will require an additional 2.3 months, a total of 7 months, and $2.33 \mathrm{~kg}$ of additional propellant for the 2.17 $\mathrm{km} / \mathrm{s}$ maneuver. The science mission orbit has a period of 4.9 hours, though higher apoapsides would be acceptable if less propellant mass than expected is available.

A highly elliptical orbit offers great flexibility for the science mission. With small burns at apoapsis, the periapsis can be raised or lowered between 80 and $200 \mathrm{~km}$ at the cost of only a few $10 \mathrm{~s} \mathrm{of} \mathrm{m} / \mathrm{s}$. This allows for deep dives and other altitude changes that could greatly improve the quality of the science product without significantly affecting the propellant budget.

Finally, after the science mission has been completed (nominally 1 Earth year or possibly after any further science extensions given that the spacecraft are still healthy), MarsCAT will transfer into a disposal orbit, raising the periapsis to $250 \mathrm{~km}$. This orbit will be stable for 100 years using worst-case-scenario drag area and drag coefficients. The amount of propellant to be held in reserve beyond the science just for this purpose is TBD; however, preliminary analysis indicates that the mission periapsis seems very stable so the reserve amount of propellant needed to raise to the disposal orbit is on the order of 10 s of grams.

\section{Structures}

\section{F. Flight System Capabilities}

Two identical 6U structures are to be custom built based on lightweight skeletonized structures designed for previous successful CubeSat mission at Texas A\&M and the University of Michigan. Provision will be made for deployable solar arrays as well as two deployable booms for the Langmuir probes. The total allowable spacecraft mass will be $14 \mathrm{~kg}$, the internal volume will be no greater than $7000 \mathrm{cc}$, the overall total dimensions will be no larger than $239.2 \mathrm{~mm}$ by $105.6 \mathrm{~mm}$ by $365 \mathrm{~mm}$, and the spacecraft will be compatible with the Planetary Systems Corporation (PSC) Canisterized Satellite Dispenser ${ }^{\mathrm{TM}}$.

2. Telecommunications (COMM)

GNC and Data Transfer. The MarsCAT CubeSat will not have access to communication with Earth until ejection from the transporting spacecraft at roughly a distance of 500,000 km from Mars. At this point there are two options for guidance, navigation and control (GNC). One option is to communicate directly to Earth (DTE) using the 
NASA Deep Space Network (DSN). The other option is to communicate with the existing Mars orbiters (e.g., MAVEN or MRO).

Communicating DTE would be done using one of the DSN 34 meter dishes (e.g, the 34 meter beam-waveguide dish) at either $\mathrm{S}$ band or $\mathrm{X}$ band. Navigation (Doppler and ranging) would require a deep-space transponder. NASA JPL has developed the Iris CubeSat-compatible deep space transponder for INSPIRE, the first CubeSat to voyage to deep space. Iris is $0.4 \mathrm{U}$ in size, $0.4 \mathrm{~kg}$ in mass, consumes $12.8 \mathrm{~W}$, and interoperates with NASA's Deep Space Network (DSN) on X-Band frequencies (7.2 GHz uplink, 8.4 GHz downlink) for command, telemetry, and navigation ${ }^{69}$. Through direct conversations with JPL, it was confirmed this system should be available commercially in the last quarter of 2015 or the first quarter of 2016. However, Iris is expected to be quite expensive. The estimated cost is about $\$ 497 \mathrm{k}$ (based on conversations with JPL), and two of them would be needed, one for each of the two 6U MarsCAT CubeSats. If Iris were to be upgraded so that it could communicate at UHF with the Electra system on the Mars orbiters using the Proximity-1 protocol, the cost would be significantly more. Iris is built on a "slice" architecture, and NASA JPL is adding a UHF receive slice now to Iris, but at present no UHF transmit slice has been added. This situation makes the proposition of adding UHF transmit and receive costly. Furthermore, since the MarsCAT CubeSats will only have low-gain antennas (LGAs), the data rate using DTE communication will be limited to a few bps at this range, based on published Iris data ${ }^{62}$. This data rate will allow for limited GNC. It will not allow for data transfer from MarsCAT to Earth at a rate that is sufficient to accommodate the science experiments. Therefore, communication with the Mars orbiters is necessary even if the Iris transponder is incorporated into MarsCAT. Therefore, the decision is to forgo the use of a deep space transponder and rely only on communication with the orbiters.

Communication with the existing Mars orbiters will be done via the UHF Electra system onboard the orbiters. The Electra system is a software defined transceiver radio system operating in the $390-450 \mathrm{MHz}$ band that uses the CCSDS Proximity-1 protocol for communication. Existing software defined radios developed for CubeSats such as the Astronautical Development LLC Li-1 radio or the L-3 CSW Cadet radio do not have Proximity-1 capability. Therefore, this capability will need to be added to the UHF transceiver radio that is selected. It is estimated that this upgrade cost will be between $\$ 125 \mathrm{k}$ and $\$ 250 \mathrm{k}$ if done by the supplier of the radio. The cost is estimated to be only about $\$ 50 \mathrm{k}$ if done in-house using student developers. However, the risk associated with this in-house upgrade to the radio is moderate. Therefore, the cost of the vendor upgrade was included in our SIMPLeX proposal as a risk reduction element. The capability to use the Electra system for GNC has already been demonstrated, though not for CubeSats. Therefore, this choice presents a low-moderate risk.

Link Budget and Data Transfer: Based on the bit error rate (BER) performance of Electra, a link budget analysis determines the communication data rate between the MarsCAT CubeSat and the Electra system on the orbiters. The results, based on published Electra data ${ }^{70}$, are shown in Table 3. The link budget has assumed a full duplex mode (8.5 W transmit) using a UHF transmit CubeSat antenna with a gain of $6 \mathrm{dBi}$ and a UHF low-gain nearly omnidirectional receive antenna (e.g., a quadrifilar helix) on the orbiter with a gain of $0 \mathrm{dBi}$. Three different BER were assumed. The range calculations for data rates at $8000 \mathrm{bps}$ and above were based directly on the published Electra data while the range calculation for data rates below this were based on extrapolation, assuming that the data rate is proportional to the SNR.

Table 3 also shows that early navigation using a data rate of a few bps can be established at a distance of about $500,000 \mathrm{~km}$ from the orbiters, which is the planned range for initial communication.

A moderate data rate $(50 \mathrm{kbps})$ will require the CubeSat to be within approximately $3,000 \mathrm{~km}$ of a relay orbiter. This data rate will be used for relaying data back to Earth from the science experiments on the MarsCAT, once MarsCAT is close to Mars in final orbit.

\begin{tabular}{|l|l|l|l|}
\hline Data Rate (bps) & \multicolumn{3}{|l|}{ Range $(\mathrm{km})$} \\
\hline & BER $=10^{-3}$ & BER $=10^{-4}$ & BER $=10^{-5}$ \\
\hline $1,024,000$ & 780 & 620 & 550 \\
\hline 512,000 & 1,400 & 1,100 & 870 \\
\hline 128,000 & 2,800 & 2,200 & 1,900 \\
\hline 32,000 & 5,500 & 4,400 & 3,900 \\
\hline 8000 & 11,000 & 8,800 & 7,800 \\
\hline 2000 & 22,000 & 17,600 & 15,500 \\
\hline 500 & 44,000 & 35,200 & 31,000 \\
\hline 125 & 88,000 & 70,400 & 62,000 \\
\hline
\end{tabular}

16

American Institute of Aeronautics and Astronautics 


\begin{tabular}{|l|l|l|l|}
\hline 31 & 176,000 & 140,800 & 124,000 \\
\hline 8 & 352,000 & 281,600 & 248,000 \\
\hline 4 & 704,000 & 563,200 & 496,000 \\
\hline
\end{tabular}

Table 3: Electra range calculation for various BER.

It is estimated that the amount of data that needs to be transmitted back to Earth from the science experiments is on the order of $100 \mathrm{MB}$ per week. If we assume an average range of about 3,000 km during the communication link, the data transfer rate will be roughly $50 \mathrm{kbps}(0.0061 \mathrm{MBs})$. It will thus take approximately 280 minutes to relay the data to the orbiter if the transfer is scheduled for once per week. If the data is transferred twice weekly, the data transfer time will be approximately 140 minutes.

The data transfer from the orbiter to Earth will be faster than that from MarsCAT to the orbiter. As an example, the MAVEN satellite transmits to Earth twice per week, for five-hour periods. The data transfer rate from MAVEN to Earth via the X-band high-gain antenna (2.1 meter dish) on MAVEN is approximately $550 \mathrm{kbps}(0.0671 \mathrm{MBs})$. Thus, it will take approximately 25 minutes to download the stored data from MAVEN to the DSN each week. If the data is transferred twice weekly, the data transfer time will be approximately 13 minutes.

MarsCAT Antennas. Each of the two 6U MarsCAT CubeSats will have a UHF antenna system onboard, which will be dual-use. The antenna system will be used for GNC and data transfer, and also for the total electron count (TEC) science experiment. The dual-use design using the same UHF bands is possible since the GNC/data transfer and the TEC experiment will not be done simultaneously. This allows for an optimum antenna system that makes the best possible use of the limited space resources on the CubeSat. Each antenna system will consist of two antennas, one on the front side of the CubeSat and one on the back side. This will allow the CubeSat to communicate with the orbiters or with the other CubeSat (for the TEC measurement) using whichever antenna has the highest received power, enabling reliable communications without having to reorient the CubeSats. This will also provide a redundancy, in case of a failure of one of the antennas. The antenna system will consist of a pair of planar dual-band UHF microstrip antennas covering the 390-405 MHz transmit and 435-450 MHz receive bands (used for both GNC and data transfer).

One of the novel aspects of the antennas is that they will be designed based on microstrip antenna technology, so that that the antennas will be planar and low profile, and can therefore be integrated directly onto the CubeSat frame, enhancing mission reliability and performance, as there will be no risk issues associated with mechanical antenna deployment. This is a significant risk factor when using conventional whip antennas on CubeSats.

At the University of Houston, the Small Satellite Laboratory recently, under NASA contract, began improving antenna design for CubeSats. Since the antenna is instrumental for communications, a failure of the antenna can have disastrous consequences for the CubeSat mission. Conventional whip antennas rely on a mechanical deployment mechanism, which is subject to jamming or breaking during deployment. One of the important goals of the SSL CubeSat research is to replace these off-the-shelf whip antennas with customized low-profile antennas that are integrated into the frame of the CubeSat body. The low-profile designs do not protrude from the CubeSat surface by more than $0.5 \mathrm{~cm}$, and are mechanically durable. There are two options for the planar dual-band UHF antenna (subsolar and transparent), and these are described below. The best candidate will be selected after examining the performance (radiation pattern, impedance match, bandwidth) and final weight of each option.

Subsolar Antennas: One type of planar low-profile antenna being developed is the subsolar antenna. This consists of a microstrip antenna that goes across the entire face of a $3 \mathrm{U}$ CubeSat. The upper face of the microstrip antenna is metal, so that the solar panels can be placed on top of the antenna without degrading the antenna performance $^{71}$. The antenna radiates from its edges. The radiating edges of the microstrip antenna are aligned with the edges of the CubeSat, and hence are not blocked by the solar panels. Such antennas have been successfully developed at the UHF $434 \mathrm{MHz}$ ISM band. Fabricated prototypes are shown in Fig. 5. These designs will be extended to dual-band performance, to cover both the transmit and receive Electra bands.

The resulting antennas are low profile and integrated directly onto the CubeSat frame, giving much better reliability and compactness in the design that what can be obtained using conventional antennas that protrude from the CubeSat. This new approach to CubeSat antenna design is expected to greatly increase mission reliability.

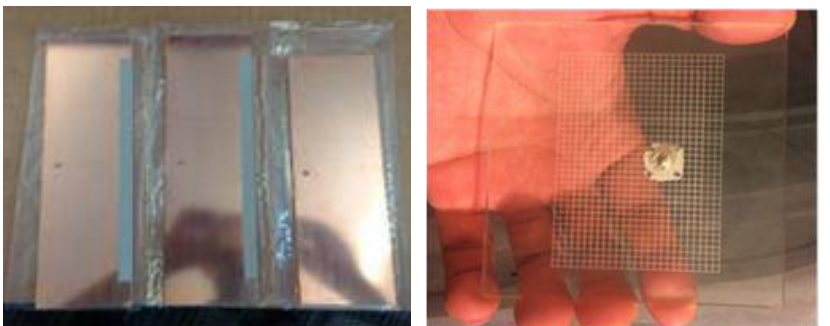

American Institute of Aeronautics and Astronautics
Figure 5. (Left) Three low-profile CubeSat Integrated Microstrip Antennas developed by Small Satellite Laboratory (before SMA connectors). (Right) Prototype low-profile 
transparent meshed microstrip patch antenna fabricated on quartz substrate.

Transparent Antennas. Transparent microstrip antennas have been developed at the SSL for operation at 2.4 $\mathrm{GHz}^{72}$. These antennas are fabricated from a meshed metal conductor (silver epoxy) that is deposited on the surface of a transparent quartz substrate. The meshed silver epoxy is used to construct both the microstrip patch antenna surface as well as the ground plane surface below the patch. Figure 5 shows one fabricated prototype.

These transparent antennas are designed to be placed on top of the solar panels on the CubeSat, so that they are functional without blocking a significant portion of the light from reaching the solar panels. A typical transparency is about $75 \%$.

These designs will be extended to cover UHF Electra bands, allowing for both communication and navigation with the Electra system on the orbiters as well as the TEC experiment.

Using these antennas, there is a mild loss of gain due to the $25 \%$ blockage, corresponding to a loss of approximately $1.2 \mathrm{~dB}$. However, there may be advantages to the transparent design in terms of the pattern or bandwidth. These antennas are not constrained to radiate only from the edges, as there is no concern about components being placed on top of the antenna, so the entire antenna surface can be used as the radiating surface, allowing for more flexible design.

A careful comparison between the two antenna types will be made to determine the best candidate for the MarCAT mission. This trade study includes a comparison of weight, reliability, radiation pattern, gain, impedance match and bandwidth.

\section{Thermal and Radiation}

The main thermal concerns are for the batteries and fuel in transit. Unsteady finite-element thermal modeling will be accomplished, and passive thermal control will be applied via judicious component placement, radiators, and thermal blankets. Once in Mars orbit, if active heating is required, simple patch heaters will be used.

At Mars, solar energetic protons (SEP) are the primary source of radiation. The on-board computer selected is rad-hardened (see Command \& Data Handling, below). As well, various of the other components, many commercially available and CubeSat compatible, will be rad tolerant or hardened.

4. Electrical Power System (EPS)

Power Budget Summary

\begin{tabular}{|c|c|c|c|c|c|c|c|}
\hline \multicolumn{2}{|l|}{ Mars Orbit Insertion } & Duration: & 7 Months & Nominal Science Orbit ${ }^{1}$ & Duration: & $17620 \mathrm{~s}$ & \\
\hline \multirow{2}{*}{\multicolumn{2}{|c|}{$\begin{array}{r}\text { MOI Consumption } \\
\text { Generation }\end{array}$}} & $31.31 \mathrm{~W}$ & $6.54 \mathrm{kWh}$ & Orbit Consumption & $9.29 \mathrm{~W}$ & $45.47 \mathrm{Wh}$ & \\
\hline & & $40.00 \mathrm{~W}$ & Direct & Generation/Battery 20\% DOD Cap & $40.00 \mathrm{~W}$ & $14.21 \mathrm{Wh}$ & \\
\hline \multirow{3}{*}{\multicolumn{2}{|c|}{ Margin }} & $8.69 \mathrm{w}$ & - & Worst Case Eclipse Demand & $10.29 \mathrm{~W}$ & $5.20 \mathrm{Wh}$ & \\
\hline & & $27.7 \%$ & - & Margin & $30.709 \mathrm{w}$ & $9.01 \mathrm{Wh}$ & \\
\hline & & & & & $43.2 \%$ & $173.1 \%$ & \\
\hline \multicolumn{2}{|l|}{ Active Comm Link ${ }^{2}$} & Duration: & $10750 \mathrm{~s}$ & & & & $\begin{array}{l}\text { Table 4: } \\
\text { Power and }\end{array}$ \\
\hline \multirow{2}{*}{\multicolumn{2}{|c|}{$\begin{array}{r}\text { Link Duration Consumption } \\
\text { Generation/Battery 20\% DOD Cap }\end{array}$}} & $23.93 \mathrm{~W}$ & $71.46 \mathrm{Wh}$ & & & & energy budget. \\
\hline & & $40.00 \mathrm{~W}$ & $14.21 \mathrm{Wh}$ & & & & \\
\hline \multicolumn{2}{|c|}{ Worst Case Eclipse Demand } & $24.93 \mathrm{~W}$ & $12.60 \mathrm{Wh}$ & & & & \\
\hline \multirow{2}{*}{\multicolumn{2}{|c|}{ Margin }} & $16.069 \mathrm{w}$ & $1.60 \mathrm{Wh}$ & & & & \\
\hline & & $22.6 \%$ & $12.7 \%$ & & & & \\
\hline \multicolumn{8}{|l|}{ Notes: } \\
\hline $\begin{array}{l}\text { 1. Worst case eclipse } \\
\text { condition over } 1 \text { Earth } \\
\text { year based on STK }\end{array}$ & \multicolumn{2}{|c|}{$\begin{array}{l}\text { 2. Link duration based on } \\
\text { average Mars Relay link } \\
\text { duration over } 1 \text { year }<2000\end{array}$} & & & & & \\
\hline eclipse data & $\mathrm{km} \mathrm{ra}$ & & & & & & \\
\hline
\end{tabular}

The MarsCAT power system consists of a $7.2 \mathrm{~V}$ bus regulated to $5 \mathrm{~V}$ and $3.3 \mathrm{~V}$ supplies. Primary power comes from a deployable solar array coupled with body-mounted cells. 185 SpectroLab XTJ triple-junction solar cells with $29.5 \%$ beginning-of-life collection efficiency will be used. Solar arrays are sized for performance after three years in the vicinity of Mars. MarsCAT is launched fully charged to provide all of the necessary start-up power required to deploy the solar arrays and fire the CAT. Testing will confirm the batteries' ability to hold charge during Mars transit. In addition, the team will investigate the prospect of a power line through the PPOD for battery maintenance.

While MarsCAT is stowed, the power system is inhibited via two redundant separation switches contacting the rear of the deployer. Upon deployment the plunger switches are released, and the power system turns on the flight computer to begin its boot cycle.

18

American Institute of Aeronautics and Astronautics 
Power Generation Degradation. Assuming coverslide degradation at $\sim 15 \%$ per $1 \mathrm{e}+161-\mathrm{MeV}$ equivalent electrons (though at Mars, solar energetic protons (SEP) are the primary source of radiation), the CREME-96 short term (solar event) predicts an equivalent $1-\mathrm{MeV}$ fluence of $6.64 \mathrm{e}+15$ for one year at $1.5 \mathrm{AU}$, or approximately $10 \%$ degradation per year for solar events similar to the October 1989 event. The long term proton fluence given by the ESP-PSYCHIC model suggests degradation on the order of an additional $0.02 \%$ per year.

Battery Sizing. Battery packs will be built from eight 3.6 V Panasonic Li-ion batteries with specific energy density of $243 \mathrm{~W} \mathrm{hr} \mathrm{kg}-1$. Two batteries will be in series with 4 sets in parallel.

Regulation and Control. The power board also provides fault protection that can be reset via the Command and Control board.

Power and Energy Budget. The power and energy budgets are shown in Table 4.

5. Propulsion (CAT)

The MarsCAT spacecraft will be propelled using the CubeSat Ambipolar Thruster (CAT), a permanent magnet helicon plasma thruster specifically designed for CubeSats. With an Isp of 690s and a thrust to power ratio of 73 $\mathrm{mN} / \mathrm{kW}$, CAT is being developed at the University of Michigan which has secured a flight to demonstrate this technology in 2016.

The MarsCAT mission will build off of the success of the upcoming CAT demonstration mission. With lessons learned the thruster will be improved to ensure mission success. The CAT demonstration mission will use xenon as a propellant but to achieve the necessary delta-v for MarsCAT without taking up too much volume in the spacecraft iodine must be used. CAT's electrodeless design is uniquely positioned to utilize iodine, which stores as a solid, minimizing propellant volume. Iodine, however, has never been demonstrated as a propellant and the TRL of the propellant management system is quite low $(\sim 2)$. Since iodine propellant is an enabling technology for this mission, so part of the MarsCAT budget will be dedicated to developing a fully functional and flight ready iodine propellant management system.

6. Guidance \& Navigation

The attitude determination and control system (ADCS) will be composed of two subsystems: the attitude estimation subsystem and the attitude control subsystem. ADCS comprises three reaction wheels (Sinclair Interplanetary RW3-0.06-28), two horizon sensors, one star tracker (Sinclair Interplanetary ST-16RT), photodiodes on the solar array for coarse sun sensing, and rate gyros all coupled with algorithms and small burns by CAT. An Accion Max-1 thruster system will be used to desaturate the wheels. Refinements on accuracy and knowledge estimates will occur once the award is in place, but it is apparent from our investigations thus far that CubeSat compatible systems are available to meet mission performance needs.

Sensor data will be fused together using a multiplicative extended Kalamn filter (MEFK), a well know and robust attitude estimation algorithm. The MEKF will have various modes whereby different sensors will be used should some not be available or fail prematurely. The attitude estimate computed using the EKF will be passed to the attitude control system. A quaternion-based filtered PID+ control law will realize both coarse and fine pointing. Rather than using a constant gain PID control law, filtered PID control plus feedforward control given the desired spacecraft attitude will be used to realize faster slewing, reduce overshoot, and a faster settling time. Filtered PID+ control is a well established, robust, control technique.

7. Command \& Data Handling $(C \& D H)$

For mitigation of radiation effects, the on-board computer will be the Space Micro rad-hard Proton $200 \mathrm{k}^{\mathrm{TM}}$ Lite FMB model for a 2-5 year mission. Advertised features include radiation hardening utilizing Space Micro's patented radiation mitigation technologies; 200 grams mass; low power for CubeSat missions (1.5W standard); optimized processing speed of 900 MFLOPS; DSP processor; Memory of 512 Mbyte SDRAM w/EDAC, 1 Mbyte EEPROM to 8 Mbyte (option), $8 \mathrm{~Gb}$ RH Flash (option); Operating System and Software Support: TI DSP/BIOS RTOS (option), TI Code Composer Studio (option), JTAG debugging support; and CubeSat PPOD compatible.

Based on the success and established framework of the AggieSat Lab at Texas A\&M, the architecture for flight software will be distributed across different, multi-threaded processes. The processing workload will be split-up among the subsystems: C\&DH, ADCS, COMM, EPS, and each of scientific payloads including CAT. Each process has its own defined hardware connections to different ports on the flight computer, and then communicates to each other.

The version control for flight code uses backups on a subversion server on a main Sun server with the AggieSat Lab at Texas A\&M. Current working versions of the software are kept on the uncontrolled portion of the server. As for test plan, some milestones include automated system checkout routine (system health) and running through automated scripts in Day-In-Life-Testing. 


\section{Conclusion}

The MarsCAT Mission is a proposed CubeSat mission to Mars, consisting of two 6U CubeSats equipped with on-board electric propulsion. The mission science goals are aimed at increasing understanding of the ionosphere of Mars, its structure and its dynamics. The mission engineering goals are to demonstrate that it is possible to operate CubeSats in Mars orbit.

\section{Acknowledgments}

We thank all of our parent institutions for their proposal development support.

\section{References}

\footnotetext{
${ }^{1}$ Haider, S. A., K. K. Mahajan, and E. Kallio, Mars ionosphere: A review of experimental results and modeling studies, Rev. Geophys., 49, RG4001, doi:10.1029/2011RG000357, 2011.

2 Withers, Paul. A review of observed variability in the dayside ionosphere of Mars. Advances in Space Research 44, no. 3, 277-307, 2009.

${ }^{3}$ Withers, P. et al., The ionosphere of Mars and its importance for climate evolution, Community white paper submitted to the 2011 Planetary Science Decadal Survey, 2011.

$\checkmark{ }^{4}$ Kliore, A. J., et al., Occultation experiment: Results of the first direct measurement of Mars's atmosphere and ionosphere. Science, 149(3689), 1243-1248, 1965.

$\checkmark 5$ Kliore, A. J., G. Fjeldbo, B. L. Seidel, M. J. Sykes, and P. M. Woiceshyn, S band radio occultation measurements of the atmosphere and topography of Mars with Mariner 9: Extended mission coverage of polar and intermediate latitudes, J. Geophys. Res., 78(20), 4331-4351, doi:10.1029/JB078i020p04331, 1973.
}

6 Nielsen, Erling, Mars Express and MARSIS, Space Science Reviews, 111, 1-2, 245-262, doi: 10.1023/B:SPAC.0000032712.05204.5e, 2004.

${ }^{7}$ Nielsen, E., H. Zou, D. A. Gurnett, D. L. Kirchner, D. D. Morgan, R. Huff, R. Orosei, A. Safaeinili, J. J. Plaut, and G. Picardi. "Observations of vertical reflections from the topside Martian ionosphere." In The Mars Plasma Environment, pp. 373388. Springer New York, 2007a.

${ }^{8}$ Hanson, W. B., S. Sanatani, and D. R. Zuccaro, The Martian ionosphere as observed by the Viking retarding potential analyzers, J. Geophys. Res., 82(28), 4351-4363, doi:10.1029/JS082i028p04351, 1977.

$\checkmark$ Nair, H., Allen, M., Anbar, A. D., Yung, Y. L., and Clancy, R. R., "A photochemical model of the Martian atmosphere," Icarus, Vol. 111, 1994, pp. 124-150.

${ }^{10}$ Krymskii, A. M., T. K. Breus, N. F. Ness, D. P. Hinson, and D. I. Bojkov, Effect of crustal magnetic fields on the near terminator ionosphere at Mars: Comparison of in situ magnetic field measurements with the data of radio science experiments on board Mars Global Surveyor, J. Geophys. Res., 108, 1431, doi:10.1029/2002JA009662, A12, 2003.

$\checkmark{ }^{11}$ Mahajan, K. K., Singh, S., Kumar, A. K., Raghuvanshi, S., and Haider, S.A., "Mars Global Surveyor radio science electron density profiles: Some anomalous features in the Martian ionosphere," J. Geophys. Res. Planets, Vol. 112, 2007 , pp. 1-11.

${ }^{12}$ Nielsen, E., X.-D. Wang, D. A. Gurnett, D. L. Kirchner, R. Huff, R. Orosei, A. Safaeinili, J. J. Plaut, and G. Picardi, Vertical sheets of dense plasma in the topside Martian ionosphere, J. Geophys. Res., 112, E02003, doi:10.1029/2006JE002723, 2007b.

${ }^{13}$ Acuña, M. H., et al., Magnetic field of Mars: Summary of results from the aerobraking and mapping orbits, J. Geophys. Res., 106(E10), 23403-23417, doi:10.1029/2000JE001404, 2001.

${ }^{14}$ Mitchell, D. L., R. P. Lin, C. Mazelle, H. Rème, P. A. Cloutier, J. E. P. Connerney, M. H. Acuña, and N. F. Ness, Probing Mars' crustal magnetic field and ionosphere with the MGS Electron Reflectometer, J. Geophys. Res., 106(E10), 23419-23427, doi:10.1029/2000JE001435, 2001.

${ }^{15}$ Harnett, E. M., and R. M. Winglee, The influence of a mini-magnetopause on the magnetic pileup boundary at Mars, Geophys. Res. Lett., 30, 2074, doi:10.1029/2003GL017852, 20, 2003.

${ }^{16}$ Harnett, E. M., and R. M. Winglee, Three-dimensional fluid simulations of plasma asymmetries in the Martian magnetotail caused by the magnetic anomalies, J. Geophys. Res., 110, A07226, doi:10.1029/2003JA010315, 2005.

${ }^{17}$ Harnett, E. M., and R. M. Winglee, Three-dimensional multi-fluid simulations of ionospheric loss at Mars from nominal solar wind conditions to magnetic cloud events, J. Geophys.Res., 111, A09213, doi:10.1029/1999JE001069, 2006.

${ }^{18}$ Brain, D. A., J. S. Halekas, R. Lillis, D. L. Mitchell, R. P. Lin, and D. H. Crider, Variability of the altitude of the Martian sheath, Geophys. Res. Lett., 32, L18203, doi:10.1029/2005GL023126, 2005.

${ }^{19}$ Dong, C. et al. (2015), Plasma and wave properties downstream of Martian bow shock: Hybrid simulations and MAVEN observations, in AAS/AGU Triennial Earth-Sun Summit, vol. 1, p. 30502.

${ }^{20}$ Brace, L. H., R. F. Theis, and W. R. Hoegy. Plasma clouds above the ionopause of Venus and their implications. Planetary and Space Science 30, 1, 29-37, 1982. 
${ }^{21}$ Russell, C. T., et al., Magnetic field and plasma wave observations in a plasma cloud at Venus. Geophysical Research Letters, 9(1), 45-48, 1982.

${ }^{22}$ Brace, L. H., R. F. Theis, W. R. Hoegy, J. H. Wolfe, J. D. Mihalov, C. T. Russell, R. C. Elphic, and A. F. Nagy, The dynamic behavior of the Venus ionosphere in response to solar wind interactions, J. Geophys. Res., 85(A13), 7663-7678, doi:10.1029/JA085iA13p07663, 1980

${ }^{23}$ Russell, C. T., and R. C. Elphic, ISEE observations of flux transfer events at the dayside magnetopause, Geophys. Res. Lett., 6(1), 33-36, doi:10.1029/GL006i001p00033, 1979.

${ }^{24}$ Wolff, R. S., B. E. Goldstein, and C. M. Yeates, The onset and development of Kelvin-Helmholtz instability at the Venus ionopause, J. Geophys. Res., 85(A13), 7697-7707, doi:10.1029/JA085iA13p07697, 1980.

$\checkmark{ }^{25}$ Russell, C. T., R. N. Singh, J. G. Luhmann, R. C. Elphic, and L. H. Brace. Waves on the subsolar ionopause of Venus. Advances in Space Research 7(12), 115-118, 1987.

${ }^{26}$ Dreher, J., G. T. Birk, K. Schindler, and A. Otto, Role of magnetic reconnection in Venus ionopause activity, J. Geophys. Res., 100(A8), 14833-14841, doi:10.1029/95JA00522, 1995.

${ }^{27}$ Ma, Y., A. F. Nagy, K. C. Hansen, D. L. DeZeeuw, T. I. Gombosi, and K. G. Powell, Three-dimensional multispecies MHD studies of the solar wind interaction with Mars in the presence of crustal fields, J. Geophys. Res., 107(A10), 1282, doi:10.1029/2002JA009293, 2002.

${ }^{28}$ Harnett, E. M., High-resolution multifluid simulations of flux ropes in the Martian magnetosphere, J. Geophys. Res., 114, A01208, doi:10.1029/2008JA013648, 2009.

${ }^{29}$ Brain, D. A., A. H. Baker, J. Briggs, J. P. Eastwood, J. S. Halekas, and T.-D. Phan, Episodic detachment of Martian crustal magnetic fields leading to bulk atmospheric plasma escape, Geophys. Res. Lett., 37, L14108, doi:10.1029/2010GL043916, 2010.

${ }^{30}$ Duru, F., D. A. Gurnett, R. A. Frahm, J. D. Winningham, D. D. Morgan, and G. G. Howes, Steep, transient density gradients in the Martian ionosphere similar to the ionopause at Venus, J. Geophys. Res., 114, A12310, doi:10.1029/2009JA014711, 2009.

${ }^{31}$ Bertucci, C., C. Mazelle, and M. Acuña. Structure and variability of the Martian magnetic pileup boundary and bow shock from MGS MAG/ER observations. Advances in Space Research 36, no. 11, 2066-2076, 2005.

32 Brain, D. A., et al., Variability of the altitude of the Martian sheath, Geophys. Res. Lett., 32, L18203, doi:10.1029/2005GL023126, 2003.

$\checkmark{ }^{33}$ Nagy, A. F., D. Winterhalter, K. Sauer, T. E. Cravens, S. Brecht, C. Mazelle, D. Crider et al., The plasma environment of Mars, In Mars' Magnetism and Its Interaction with the Solar Wind, pp. 33-114. Springer Netherlands, 2004.

$\checkmark{ }^{34}$ Eastwood, J. P., D. A. Brain, J. S. Halekas, J. F. Drake, T. D. Phan, M. Øieroset, D. L. Mitchell, R. P. Lin, and M. Acuña, Evidence for collisionless magnetic reconnection at Mars, Geophys. Res. Lett., 35, L02106, doi:10.1029/2007GL032289, 2008.

${ }^{35}$ Pope, S. A., M. A. Balikhin, T. L. Zhang, A. O. Fedorov, M. Gedalin, and S. Barabash, Giant vortices lead to ion escape from Venus and re-distribution of plasma in the ionosphere, Geophys. Res. Lett., 36, L07202, doi:10.1029/2008GL036977, 2009.

${ }^{36}$ Terada, N., S. Machida, and H. Shinagawa, Global hybrid simulation of the Kelvin-Helmholtz instability at the Venus ionopause, J. Geophys. Res., 107(A12), 1471, doi:10.1029/2001JA009224, 2002.

${ }^{37}$ Lilensten, J. Bernard, D., Barthélemy, M., Gronoff, G., Wedlund, C. Simon and Opitz, A., "Prediction of blue, red and green aurorae at Mars," Planetary and Space Science, May 2015, PII : S0032-0633(15)00130-0, DOI : 10.1016/j.pss.2015.04.015

${ }^{38}$ González-Galindo, F., Chaufray, J.-Y., Lopez-Valverde, M. A., Gilli, G., Forget, F., Leblanc, F., Modolo, R., Hess, S., and Yagi, M., "Three-dimensional Martian ionosphere model: I. The photochemical ionosphere below 180 km," J. Geophys. Res. Planets, Vol. 118, 2013, pp. 2105-2123.

${ }^{39}$ Chaufray, J. -Y., Gonzáles-Galindo, F., Forget, F., Lopez-Valverde, M., Leblanc, F., Modolo, R., Hess, S., Yagi, M., Blelly, P.-L., and Witasse, O., "Three-dimensional Martian ionosphere model: II. Effect of transport processes due to pressure gradient," J. Geophys. Res. Planets, Vol. 119, 2014, pp.1614-1636.

${ }^{40}$ Allen, M., Yung, Y. L., and Waters, J. W., "Vertical transport and photochemistry in the terrestrial mesosphere and lower thermosphere (50-120 km)," J. Geophys. Res., Vol. 86, 1981, 3617-3627.

${ }^{41}$ Yung, Y. L., Allen, M., and Pinto, J. P., "Photochemistry of the atmosphere of Titan - Comparison between model and observations," Astrophys. J. Suppl., Vol. 55, 1984, pp. 465-506.

${ }^{42}$ Gladstone, G. R., Allen, M., Yung, Y. L., "Hydrocarbon photochemistry in the upper atmosphere of Jupiter," Icarus, Vol. 119, 1996, pp. 1-52.

${ }^{43}$ Liang, M. C., Shia, R.-L., Lee, A. Y.-T., Allen, M., Friedson, A. J., Yung, Y. L., "Meridional transport in the stratosphere of Jupiter," Astrophysical Journal, Vol. 635, 2005, pp. L177-L180.

$\checkmark{ }^{44}$ Shia, R. L., Ha, Y. L., Wen, J.-S., Yung, Y. L., "Two-Dimensional atmospheric transport and chemistry model - numerical experiments with a new advection algorithm," J. Geophys. Res. Atmospheres, Vol. 95, 1990, pp. 7467-7483.

${ }^{45}$ Richardson, M. I., Toigo, A. D., Newman, C. E., "PlanetWRF: A general purpose, local to global numerical model for planetary atmospheric and climate dynamics," J. Geophys. Res. Planets, Vol. 112, 2007, pp. 1-29.

${ }^{46}$ Toigo, A. D., Lee, C., Newman, C. E., Richardson, M. I., "The impact of resolution on the dynamics of the martian global atmosphere: Varying resolution studies with the MarsWRF GCM," Icarus, Vol. 221, 2012, pp. 276-288. 
${ }^{47}$ Crider, D. H., J. Espley, D. A. Brain, D. L. Mitchell, J. E. P. Connerney, and M. H. Acuña, Mars Global Surveyor observations of the Halloween 2003 solar superstorm's encounter with Mars, J. Geophys. Res., 110, A09S21, doi:10.1029/2004JA010881, 2005.

${ }^{48}$ Morgan, D. D., et al., Effects of a strong ICME on the Martian ionosphere as detected by Mars Express and Mars Odyssey, J. Geophys. Res. Space Physics, 119, 5891-5908, doi:10.1002/2013JA019522, 2014.

49 Trotignon, J. G., E. Dubinin, R. Grard, S. Barabash, and R. Lundin, Martian planetopause as seen by the plasma wave system onboard Phobos 2, J. Geophys. Res., 101(A11), 24965-24977, doi:10.1029/96JA01898, 1996.

$\checkmark 50$ Collinson, G. A., et al., A survey of hot flow anomalies at Venus, J. Geophys. Res. Space Physics, 119, 978-991, doi:10.1002/2013JA018863, 2014.

${ }^{51}$ Vignes, D. et al., The solar wind interaction with Mars: Locations and shapes of the bow shock and the magnetic pile-up boundary from the observations of the MAG/ER Experiment onboard Mars Global Surveyor, Geophysical Research Letters, 27, 49-52, DOI: 10.1029/1999GL010703, 2000.

${ }^{52}$ Luhmann, J. G., W. T. Kasprzak, and C. T. Russell, Space weather at Venus and its potential consequences for atmosphere evolution, J. Geophys. Res., 112, E04S10, doi:10.1029/2006JE002820, 2007.

${ }^{53}$ Edberg, N. J. T., et al., Atmospheric erosion of Venus during stormy space weather, J. Geophys. Res., 116, A09308, doi:10.1029/2011JA016749, 2011.

54 Cal Poly CubeSat and Poly Picosat Orbital Deployer (PPOD) standards (http://cubesat.calpoly.edu/index.php/documents/developer ), with the Launch Services Program (LSP) Program Level Dispenser and CubeSat Requirements Document (LSP-REQ-317.01 Revision B)

${ }^{55}$ Hevner, Holemans, Puig-Suari, Twiggs, An Advanced Standard for Cubesats; related to 6U and larger, SSC11-II-3, 25 ${ }^{\text {th }}$ Annual AIAA/USU Conference on Small Satellites. 2011.

${ }^{56}$ Leuzinger, A., and A. Taylor, Magneto-Inductive Technology Overview, http://www.pnicorp.com/technology/papers (accessed August 28, 2014), 2010.

57 Allen, Lindsay, Ariane Chepko, Theodore Beach, and Odile Clavier, Algorithms for Accurate LEO Geomagnetic Measurements with Satellite-Mounted Magnetometers, IEEE Transactions on Aerospace and Electronic Systems, 2014

${ }^{58}$ Sheinker, A. and M. B. Moldwin, Adaptive Interference Cancelation using a Pair of Magnetometers, submitted to IEEE Transactions on Aerospace and Electronic Systems, 2015.

${ }^{59}$ Vasyliunas, Vytenis M. "12. Deep Space Plasma Measurements." Methods in Experimental Physics 9 (1971): 49-88.

${ }^{60}$ Gloeckler, George. "Ion composition measurement techniques for space plasmas." Review of scientific instruments 61, no. 11 (1990): 3613-3620.

${ }^{61}$ Kasper, J. C., A. J. Lazarus, J. T. Steinberg, K. W. Ogilvie, and A. Szabo. "Physics-based tests to identify the accuracy of solar wind ion measurements: A case study with the Wind Faraday Cups." Journal of Geophysical Research: Space Physics (1978-2012) 111, no. A3 (2006).

${ }^{62}$ Hershkowitz, Noah. "How Langmuir probes work." Plasma Diagnostics 1 edited by O Auicello and D L Flamm, Academic Press, New York pp113-183, 1989.

${ }^{63}$ Lieberman MA, Lichtenberg AJ. Principles of plasma discharges and material processing: MRS Bull. 30 899; 2005.

${ }^{64}$ Longmier, B. W., E. A. Bering, III, M. D. Carter, L. D. Cassady, W. J. Chancery, F. R. Chang Díaz, T. W. Glover, N. Hershkowitz, A. V. Ilin, G. E. McKaskill, C. S. Olsen and J. P. Squire, Ambipolar ion acceleration in an expanding magnetic nozzle, Plasma Sources Sci. Technol., 20, 015007, doi: 10.1088/0963-0252/20/1/015007, 2011.

${ }^{65}$ Longmier BW. Plasma Sheath Behavior in a Total Nonambipolar Radio Frequency Generated Plasma Electron Source. Ph.D. dissertation, University of Wisconsin, Madison, WI, 2007.

${ }^{66}$ Fish, C. S., C. M. Swenson, G. Crowley, A. Barjatya, T. Neilsen, J. Gunther, I. Azeem et al. "Design, Development, Implementation, and On-orbit Performance of the Dynamic Ionosphere CubeSat Experiment Mission." Space Science Reviews 181, 61-120, 2014.

$\checkmark 67$ Yizengaw, Endawoke, M. B. Moldwin, Peter Lawrence Dyson, and E. A. Essex. "Using tomography of GPS TEC to routinely determine ionospheric average electron density profiles." Journal of Atmospheric and Solar-Terrestrial Physics 69, no. 3 (2007): 314-321.

${ }^{68}$ Ergun, R. E., D. E. Larson, T. Phan, D. Taylor, S. Bale, C. W. Carlson, I. Roth et al. "Feasibility of a multisatellite investigation of the Earth's magnetosphere with radio tomography." Journal of Geophysical Research: Space Physics (19782012) 105, no. A1 (2000): 361-373

${ }^{69}$ Duncan, Smith, and Aguirre, Iris Transponder - Communications and Navigation for Deep Space, SSC14-IX-3, 28 ${ }^{\text {th }}$ Annual AIAA/USU Conference on Small Satellites, 2014.

${ }^{70}$ Edwards C. D., Jedrey T. C., Schwartzbaum E., Devereaux E., DePaula S. R., Dapore M., and Fischer T. W., "The Electra Proximity Link Payload for Mars Relay Telecommunications and Navigation," IAC-03-Q.03.A06, Intl. Astronautical Congress 2003, Sept.-Oct. 2003.

${ }^{71}$ Montaño, R., Nicole Neveu, Simon Palacio, Elizabeth Martinez, David R. Jackson, Ji Chen, Patrick W. Fink, and Robert S. Provence. "Development of Low-profile Antennas for CubeSats." (2014). 
$>{ }^{72}$ Neveu, N., M. Garcia, J. Casana, R. Detloff, D. R. Jackson, and J. Chen. "Transparent microstrip antennas for CubeSat applications." In 2013 IEEE International Conference on Wireless for Space and Extreme Environments (WiSEE), pp. 1-4. 2013. 